

\title{
Effect of Multiscale Endplates on Wing-Tip Vortex
}

Pascal Gehlert, Ziad Cherfane, Gioacchino Cafiero, John Christos Vassilicos

\section{To cite this version:}

Pascal Gehlert, Ziad Cherfane, Gioacchino Cafiero, John Christos Vassilicos. Effect of Multiscale Endplates on Wing-Tip Vortex. AIAA Journal, 2021, 59 (5), pp.1614-1628. 10.2514/1.J059878 . hal-03146948

\section{HAL Id: hal-03146948 \\ https://hal.science/hal-03146948}

Submitted on 23 Jul 2021

HAL is a multi-disciplinary open access archive for the deposit and dissemination of scientific research documents, whether they are published or not. The documents may come from teaching and research institutions in France or abroad, or from public or private research centers.
L'archive ouverte pluridisciplinaire HAL, est destinée au dépôt et à la diffusion de documents scientifiques de niveau recherche, publiés ou non, émanant des établissements d'enseignement et de recherche français ou étrangers, des laboratoires publics ou privés. 


\title{
Effect of Multi-Scale Endplates on the Wingtip Vortex
}

\author{
Pascal Gehlert* \\ Department of Engineering, University of Cambridge, Trumpington St, CB2 1PZ Cambridge, UK \\ Ziad Cherfane $e^{\dagger}$ \\ Department of Aeronautics, Imperial College London, Exhibition Road, London, SW7 2AZ, UK
}

\author{
Gioacchino Cafiero \\ Department of Mechanical Engineering Sciences, University of Surrey, Stag Hill Campus, Guildford, GU2 7HX, UK. \\ J. Christos Vassilicos ${ }^{\S}$ \\ Univ. Lille, CNRS, ONERA, Arts et Métiers ParisTech, Centrale Lille, UMR 9014 - LMFL - Laboratoire de Mécanique \\ des fluides de Lille - Kampé de Feriet, F-59000 Lille, France
}

An experimental investigation is conducted to study the effect of multi-scale endplates on the wing tip vortex and wing aerodynamic performance at Reynolds numbers of 215000 and 244000 based on the wing mean chord of a NACA 0012 half span wing. Two groups of endplates are tested. The first group has three different fractal patterns applied along the top edge of the endplate while the second group has three different two-scale patterns applied to its outboard surface. It is experimentally shown that both geometry modifications produce a weaker and less coherent vortex. Moreover, while a peak in the power spectral density of the fluctuating axial velocity at the vortex core at Strouhal numbers between 3 and 10 is observed for the plain endplates of both groups, it vanishes for the multi-scale endplates. This suggests that the multiscale endplates act on the vortex structure making it less coherent through the production of smaller eddies as evidenced by the velocity spectra. The multi-scale endplates also show potential improvements in terms of the wing aerodynamic efficiency, with the maximum $C_{l} / C_{d}$ being up to $11.1 \%$ larger than for the corresponding plain endplate.

\section{Nomenclature}

$$
\begin{aligned}
& \alpha=\text { Angle of attack }(\mathrm{deg}) \\
& a \quad=\text { Amplitude of the fractal pattern }(\mathrm{m}) \\
& \beta \quad=90-\varphi(\mathrm{deg}) \\
& C_{d} \quad=\text { Drag coefficient }
\end{aligned}
$$

\footnotetext{
*PhD Candidate.

$\dagger \mathrm{PhD}$ Candidate.

Lecturer in Aerospace Engineering.

${ }^{\S}$ CNRS Director of Research First Class.
} 


$$
\begin{array}{ll}
C_{l} & =\text { Lift coefficient } \\
C_{r} & =\text { Root chord (m) } \\
C_{t} & =\text { Tip chord (m) } \\
D_{f} & =\text { Fractal dimension } \\
d_{w} & =\text { Diameter of the hot wire sensor }(\mathrm{m}) \\
f & =\text { Frequency }(\mathrm{Hz}) \\
\gamma & =\text { Taper ratio } \\
h & =\text { Endplate height }(\mathrm{m}) \\
\Lambda_{c} / 4 & =\text { Sweep angle at quarter chord }(\mathrm{deg}) \\
\lambda & =\text { Fractal pattern wavelength }(\mathrm{m}) \\
l_{i} & =\text { Fractal pattern lengthscale }(\mathrm{m}) \\
l_{w} & =\text { Length of the hot wire sensor }(\mathrm{m}) \\
P S D & =\text { Power spectral density }\left(\mathrm{m}^{2} / \mathrm{s}\right) \\
\mathcal{P} & =\text { Fractal pattern perimeter }(\mathrm{m}) \\
\varphi & =\text { Chevron angle of the fractal pattern }(\mathrm{deg}) \\
\rho & =\text { Density }\left(\mathrm{kg} / \mathrm{m}^{3}\right) \\
\varrho & =\text { Ratio between fractal iterations } \\
S_{m} & =\text { Manipulated surface } \\
s & =\text { Wing half-span }(\mathrm{m}) \\
u^{\prime} & =\text { Root mean square of the velocity fluctuation } \\
U_{\infty} & =\text { Freestream velocity }(\mathrm{m} / \mathrm{s}) \\
U_{t} & =\text { Transverse velocity }(\mathrm{m} / \mathrm{s}) \\
V & =\text { Velocity component aligned with } x-\text { axis }(\mathrm{m} / \mathrm{s}) \\
& =\text { Velocity component aligned with } y-\text { axis }(\mathrm{m} / \mathrm{s}) \\
& =\text { Velocy component aligned with } z-\text { axis }(\mathrm{m} / \mathrm{s})
\end{array}
$$

Subscripts

$\mathrm{i} \quad=$ Fractal iteration number

\section{Introduction}

Tip vortices are encountered in applications where a finite surface withstands a pressure difference. Typical cases are lifting wings, turbines in aero-engines and helicopter blades. In these situations, there may be the need to displace or significantly reduce the lifetime of the tip vortices, as they can hinder the performance or generate significant noise. 
In the case of aeroplanes, the natural breakdown of the tip vortex occurs due to fluid dynamic instabilities. The vortices shed from the wing tip interact with each other at a distance of roughly 40-50 wing spans behind the aircraft. Here, two main processes arise, also known as Crow [1] and Widnall [2] instabilities. These phenomena destabilize the coherent trailing vortex and cause its subsequent decay. A quicker dissipation of the wing tip vortex is of economic and environmental benefit, since it minimizes the aircraft waiting time at airports and hence fuel waste. A large body of research has focused on achieving this target. A detailed review focusing on vortices shed by aircraft and their impact on air travel safety as well as their motion and persistence is provided by Spalart [3].

Both active and passive methodologies have been used to mitigate tip vortices. Synthetic jets at the tips of the wing [4, 5] and oscillating flaps and winglets [6] are examples of the former and further extensive reviews of active methodologies are provided by Crouch [7] and Gursul \& Wang [8].

Endplates are a more conventional and widely applied solution to mitigate the tip vortex. Since their introduction in 1897 by Frederick W. Lanchester [9], they have had a substantial impact on aviation as a way to reduce the lift induced drag. In the 1970s the concept was further advanced by Richard T. Whitcomb, when he coined the term winglet to highlight the intricate aerodynamic design process of wing tip devices [10]. The main advantage of winglets, compared to wing extensions, comes when there is a restriction on the root bending moment [11]. Significant development in the design of winglet geometries has led to their frequent introduction on existing large wide-body aircraft. Only recently the B737 family was retrofitted with an innovative blended winglet design, leading to a fuel saving of $4 \%$ and a noise reduction in the airport vicinity of $6.5 \%$ [12]. It therefore seems clear that geometry modifications to the wing and endplates can have a substantial effect on the overall aircraft performance and this thus warrants their in depth research.

Fractal geometries have shown strong effects on flow field parameters, particularly on turbulence properties. Several studies have focused both on the potential applications, including heat and mass transfer enhancement [13--15], more efficient combustion using opposing jets flames [16], and the fundamental flow properties of fractal generated turbulence [17-19]. In an experimental study by Nedić et al. [20], it was shown that the $C_{l} / C_{d}$ of a NACA 0012 wing can be increased up to $8 \%$ by introducing fractal modifications to the trailing edge. The authors suggested that enhanced mixing might be the physical mechanism behind their results. They support their argument through hot wire anemometry measurements in the wing wake, where they observe a decrease in the coherence spectra measured across the wake with increasing fractal iteration.

A further experimental study by Nedić et al. [21] examined the drag and wake characteristics of square, circular, and fractal geometries; all the geometries being equivalent in terms of frontal area. Among the important results of this research was that vortex shedding energy decreased by up to $60 \%$ with increasing fractal iteration. Numerical research by Laizet \& Vassilicos [22] on fractal grids installed in channels suggested that a non-fractal grid created a higher turbulence intensity and pressure drop than a fractal grid with the same blockage ratio. Similar findings were reported by [23, 24] in the case of impinging jets with fractal generated turbulence. The common conclusion of the 
authors was that fractal geometries promote enhanced mixing.

It is therefore of interest to investigate the effect of multi-scale geometry modifications to endplates mounted on a wing model. Two different geometry manipulations are considered, resulting in two groups of endplates:

1) An increasingly higher number of fractal iterations is introduced along the tip (top edge) of the endplate. This is similar to Nedić et al. [20] where a fractal pattern was applied to the trailing edge of the wing.

2) The outboard surface of the endplate is modified to feature multi-scale geometries whilst the frontal area of the endplate (i.e. the side facing the freestream) remains constant.

For both sets of endplates the effectiveness of the geometry modification is assessed by comparing the aerodynamic coefficients to those of a plain endplate. Furthermore, the spectral analysis of the wingtip vortex provides relevant insight into the redistribution of the energy across the scales, which is a strong indicator for vortex decay.

The structure of the remainder of this paper is as follows: the experimental setup, the measurement techniques and the two groups of endplates used are detailed in section $I$ The results are then divided into two sections, where the effect of the modification to the endplate's top edge and the outboard surface are detailed in sections III.A and III.B. respectively. Finally, some common conclusions are drawn in section IV

\section{Experimental Setup}

\section{A. Facilities and Experimental Model}

The Donald Campbell wind tunnel at Imperial College London was used to conduct the experimental investigation. It consists of a test section which is $3.35 \mathrm{~m}$ long, $1.37 \mathrm{~m}$ wide and $1.22 \mathrm{~m}$ high and features a contraction ratio of $5.7: 1$. The background turbulence intensity level is $0.24 \%$ at $15 \mathrm{~ms}^{-1}$ and $0.26 \%$ at $17 \mathrm{~ms}^{-1}$.

A tapered, swept back, semi-wing based on a NACA 0012 airfoil was used. The model is the same to the one used in [25]. It was mounted at the mid height of the test section and extends from the wind tunnel walls half way into the test section, in order to reduce the interference from the walls to a minimum. The half span of the wing was $s=0.685$ $\mathrm{m}$ and it features a tip and root chord of $C_{t}=0.1 \mathrm{~m} C_{r}=0.333 \mathrm{~m}$, respectively. All the geometric parameters of the model are listed in Table 1 and a schematic representation of the wing model is provided in Figure 1 .

ABS-M30 was used to rapid prototype the wing and the surface was sanded down to achieve a smooth finish. To maintain the desired shape, two silver steel bars extend along the span in order to minimise wing deflection. Finally, any leak paths were removed by applying wax to the junction between the wing edge and the endplate.

The wing was connected to a strut located outside of the wind tunnel. This makes it possible to change the angle of attack of the wing and this was regulated using a digital inclinometer with a resolution of $0.1^{\circ}$. The wing was connected to the strut through a six-axis load cell ATI FTD-Gamma IP68 (1/80 N in plane resolution, 1/40 N out of plane resolution). Considering the wing size and the typical values of the aerodynamic forces acting on the model, the 
accuracy of the lift and drag coefficients was estimated to be $1 \%$ and $6 \%$, respectively, in the worst case scenario where the angle of attack equals zero and the aerodynamic force is therefore smallest. The accuracy on the drag coefficient reduces to $3 \%$ in the case of angle of attack equal to 6

.Whenmountingthewingitwasensuredthattherewasnobridgingbetweenthewingandthewindtunnelwalls, astoavoidanybiasi

Table 1 Wing Dimensions.

\begin{tabular}{lc}
\hline Section Geometry & NACA 0012 \\
Half Span, $s(\mathrm{~m})$ & 0.685 \\
Root Chord, $C_{r}(\mathrm{~m})$ & 0.333 \\
Tip Chord, $C_{t}(\mathrm{~m})$ & 0.1 \\
Mean aerodynamic Chord, $\bar{c}(\mathrm{~m})$ & 0.2165 \\
Taper Ratio, $\gamma$ & 0.3 \\
\hline
\end{tabular}

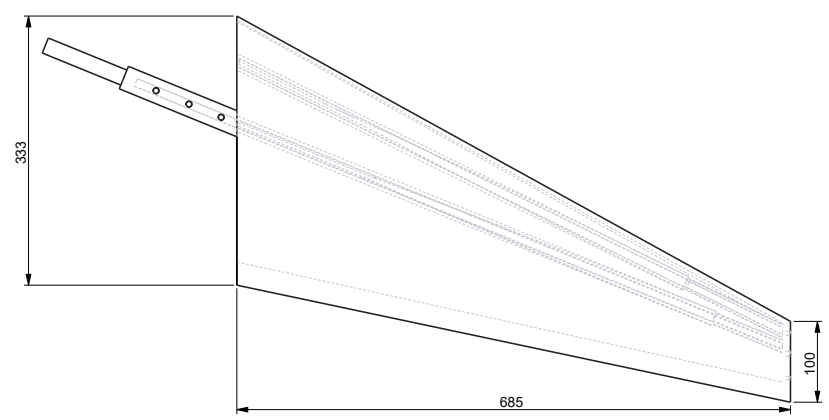

Fig. 1 Sketch of the wing from a top view. All dimensions are in $\mathbf{m m}$. The internal reinforcement bars are depicted for completeness.

\section{B. Endplate Geometries}
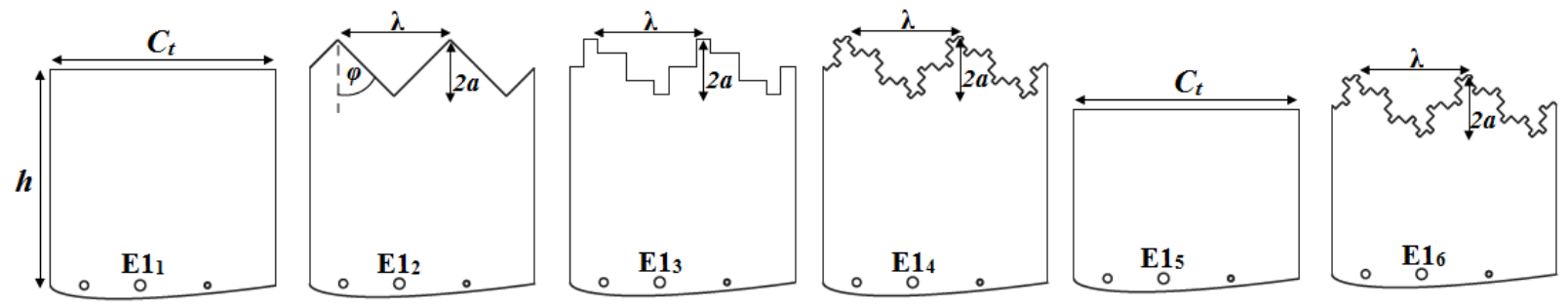

Fig. 2 Group 1 endplate geometries. $E 1_{1}-E 1_{6}$ from left to right.

The first part of this work focuses on a group of endplates where a fractal pattern is applied to the top edge. Regardless of the number of fractal iterations, the surface area of the endplate remains constant. For a fixed height $h$, four different cases are considered, $E 1_{1}$ to $E 1_{4}$, where the number of fractal iterations ranges from 0 (i.e. plain endplate) to 3. The maximum number of iterations is set by the accuracy of the laser cutting process used to manufacture the endplates as well as the structural integrity of the endplates. The effect of the endplates' height is also investigated, but 
only in the two limiting cases, where the fractal iterations are 0 and 3.

The chevron pattern is obtained by using four segments of length $l_{i}$, hence causing the number of segments that form the pattern to increase by $4^{i}$, where $i$ is the iteration number for each successive iteration.

The fractal dimension, $D_{f}$, is given by

$$
D_{f}=\frac{\ln 4^{i}}{\ln \varrho}
$$

where the ratio, $\varrho$, between successive iterations is

$$
\varrho=4 \cos \beta=\left(\frac{\lambda}{l_{i}}\right)^{1 / i}
$$

Here, $\beta=90-\varphi$ and $\varphi$ is the chevron angle.

Ultimately, the perimeter of the fractal segment, $\mathcal{P}$, can be calculated using

$$
\mathcal{P}=\frac{4^{i} l_{i} C_{t}}{\lambda}
$$

The dimensions of the endplates are summarized in Table 2 .

Table 2 Geometric details of the endplates belonging to Group $1 . D_{f}$ gives the fractal dimension, $i$ the fractal iteration, $\lambda$ the wavelength of the pattern, $l_{i}$ the segment length, $\varphi$ the chevron angle, $a$ the amplitude and $\mathcal{P} / C_{t}$ the ratio between the perimeter and the tip chord.

\begin{tabular}{ccccccccc} 
EP no. $(\mathrm{n})$ & $D_{f}$ & $i$ & $\lambda(\mathrm{mm})$ & $l_{i}(\mathrm{~mm})$ & $\varphi(\mathrm{deg})$ & $2 a(\mathrm{~mm})$ & $\mathcal{P} / C_{t}$ & $h(\mathrm{~mm})$ \\
\hline$E 1_{1}$ & - & - & - & - & - & - & 1 & 95.9 \\
$E 1_{2}$ & 1.33 & 1 & 50 & 17.68 & 45 & 25.00 & 1.41 & 95.9 \\
$E 1_{3}$ & 1.33 & 2 & 50 & 6.25 & 45 & 25.00 & 2.00 & 95.9 \\
$E 1_{4}$ & 1.33 & 3 & 50 & 2.21 & 45 & 28.13 & 2.83 & 95.9 \\
$E 1_{5}$ & - & - & - & - & - & - & 1 & 64 \\
$E 1_{6}$ & 1.33 & 3 & 50 & 2.21 & 45 & 28.13 & 2.83 & 64 \\
\hline
\end{tabular}

The second part of the investigation focuses on the effect of introducing a multi-scale pattern on the outboard surface of the endplate, as shown in Figure 3

The multi-scale pattern is applied to the outboard surface by alternatingly increasing and decreasing the endplate thickness, as to keep the frontal area constant. Two lengthscales, $l_{0}$ and $l_{1}$, are used to create the applied pattern, as shown in Figure 4. Further to this, two different parameters are considered: the ratio between the scales $\left(R_{l}\right)$ and the ratio of the manipulated endplate surface to the total outboard surface area $\left(S_{m}\right)$. Four different endplates are tested: a clean $\left(E 2_{1}\right)$ and three modified configurations $\left(E 2_{2}\right.$ to $\left.E 2_{4}\right)$. For $E 2_{2}$ and $E 2_{3}$ the multi-scale pattern is applied to the entire outboard surface, whereas for $E 2_{4}$ the multi-scale pattern is confined to the top of the endplate. The geometric details of the endplates belonging to Group 2 are listed in Table 3 . This group of endplates is also manufactured from 
ABS-M30 material using rapid prototyping.

An example of a Group 1 and Group 2 endplate mounted to the wing in the wind tunnel is shown in Figure 5.

Table 3 Geometric details of the endplates belonging to Group 2. $l_{0}$ represents the length of the first scale. $l_{1}$ represents the length of the second scale. $R_{l}=l_{1} / l_{0} . \% S_{m}$ indicates the percentage of manipulated surface with respect to the total surface area of the endplate.

\begin{tabular}{ccccc} 
EP no. $(\mathrm{n})$ & $l_{0}(\mathrm{~mm})$ & $l_{1}(\mathrm{~mm})$ & $R_{l}(\mathrm{~mm})$ & $\% S_{m}$ \\
\hline$E 2_{1}$ & - & - & - & 0 \\
$E 2_{2}$ & 2.50 & 3.00 & 1.20 & 100 \\
$E 2_{3}$ & 1.50 & 2.50 & 1.67 & 100 \\
$E 2_{4}$ & 2.50 & 3.00 & 1.20 & 7.50 \\
\hline
\end{tabular}
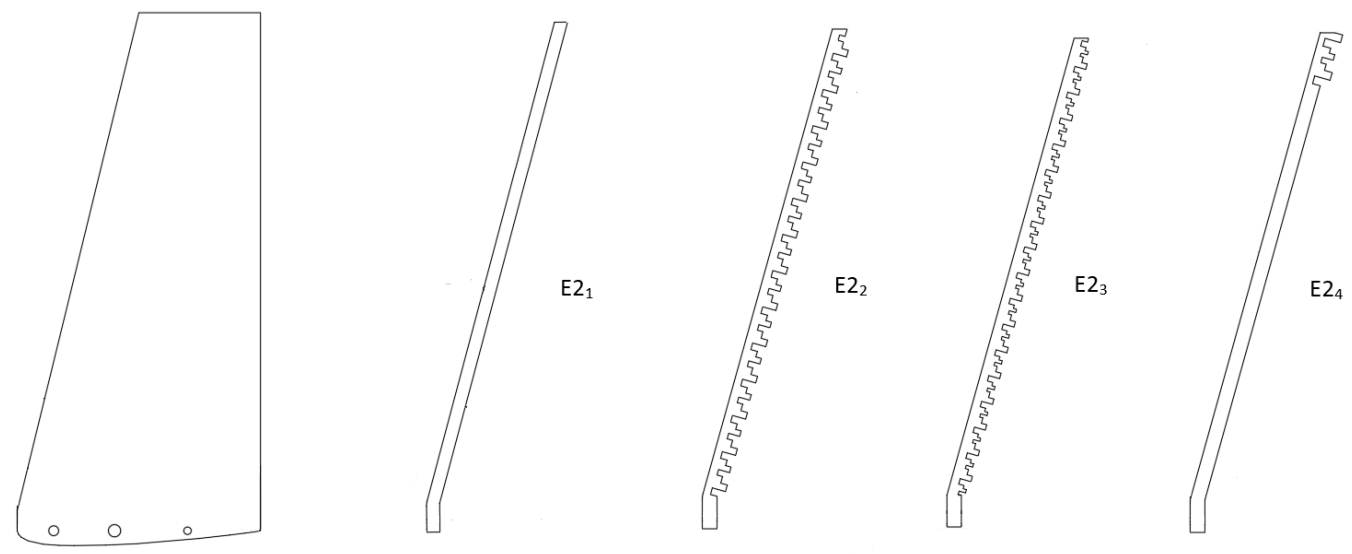

Fig. 3 Group 2 endplates geometries.

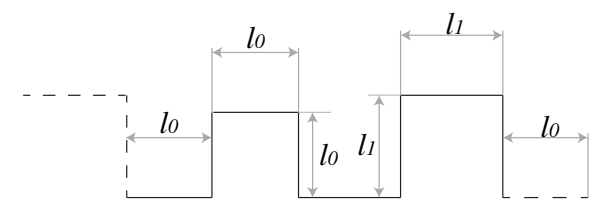

Fig. 4 Schematic representation of the Group 2 surface manipulation. A full pattern is represented using a continuous line. Dashed lines are used to indicate the continuing pattern. Values of $l_{0}$ and $l_{1}$ are listed in Table 3 .

\section{Measurement Techniques}

For all investigated cases, the reference frame is set such that the origin is located at the trailing edge of the tip of the wing, when the angle of attack, $\alpha$, is equal to $0^{\circ}$. The $x$-axis is aligned with the freestream, the $y$-axis is in the spanwise direction and points outwards, and the $z$-axis is oriented vertically. 
Force measurements, hot wire anemometry (HWA) and stereoscopic particle image velocimetry (stereo-PIV) are performed for the Group 1 endplates. All the measurements are carried out at the same Reynolds number $R e$, which is calculated based on the mean aerodynamic chord and equal to 215000. HWA and stereo-PIV experiments are performed at an angle of attack of $6^{\circ}$.

For Group 2 endplates, force measurements and HWA experiments are carried out at a slightly higher Reynolds number, $R e=244000$. The difference in Reynolds number is deemed sufficiently small as to not cause any significant changes to the flow regime.



(a) $E 1_{4}$.

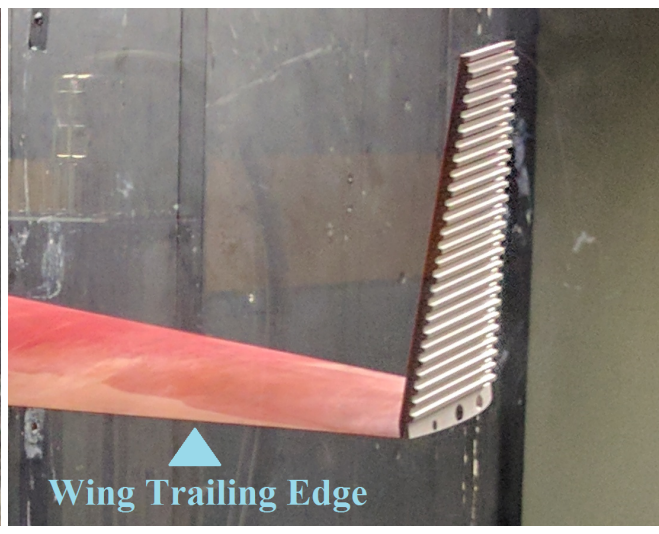

(b) $E 2_{2}$.

Fig. 5 Picture of a mounted endplate from each group.

HWA measurements were performed using a single sensor uni-directional constant temperature hot-wire probe. The sensing length, $l_{w}$, and diameter, $d_{w}$, of the horizontally orientated wire were $1 \mathrm{~mm}$ and $5 \mu \mathrm{m}$ respectively. The ratio between the sensing length and wire diameter, $l_{w} / d_{w}$, is therefore approximately 200 . The hot wire was driven by a Dantec Streamline constant temperature anemometer. Data were sampled at a frequency of $50 \mathrm{kHz}$ using a 16-bit National Instruments NI-6341 (USB) data acquisition card. Each HWA measurement lasted for $60 \mathrm{~s}$, which was shown to be sufficiently long for the convergence of the turbulent statistics studied here. The velocity spectra of the streamwise fluctuating velocity provide information about the large- and small-scale resolution of our measurements as well as about the presence of coherent/periodic structures. The computation of the velocity spectra was performed using the Welch method, by subdividing the velocity signal into 50 Hanning windows with an overlap of $75 \%$ and subsequent averaging.

An automated 3D traverse system was used to move the hot-wire probe along the $x, y$ and $z$-axis with micrometric accuracy. The traverse was located outside of the tunnel with an arm extending into the test section, to reduce any aerodynamic interference to a minimum. Furthermore, the probe was mounted using a specifically designed $a d$ hoc holder which increased the distance between the holding arm and the measurement plane.

High frequency measurements of flow structures can be obtained using hot-wires. The results are low in noise and allow for the computation of the velocity and power spectra. Moreover, statistical turbulence information can be 
obtained and low turbulence levels can be measured [26]. However, the physical presence of the probe and the probe arm cause these measurements to be intrusive. The wingtip vortex is found to be both sensitive and insensitive to this disruption in literature. Whilst vortex bursting was reported by Green et al. [27], Devenport et al. [26] and hot-wire / laser anemometer comparisons by Orloff [28] suggested that the wing-tip vortex is unaffected. The current investigation therefore took care to ensure that no disturbance, displacement or bursting of the wingtip vortex occurred. The forces acting on the wing were found to be invariant regardless of whether or not the hot-wire probe was present and a good correlation between the structure of the wingtip vortex measured using HWA and stereo-PIV further indicated that the flow field was not unduly affected.

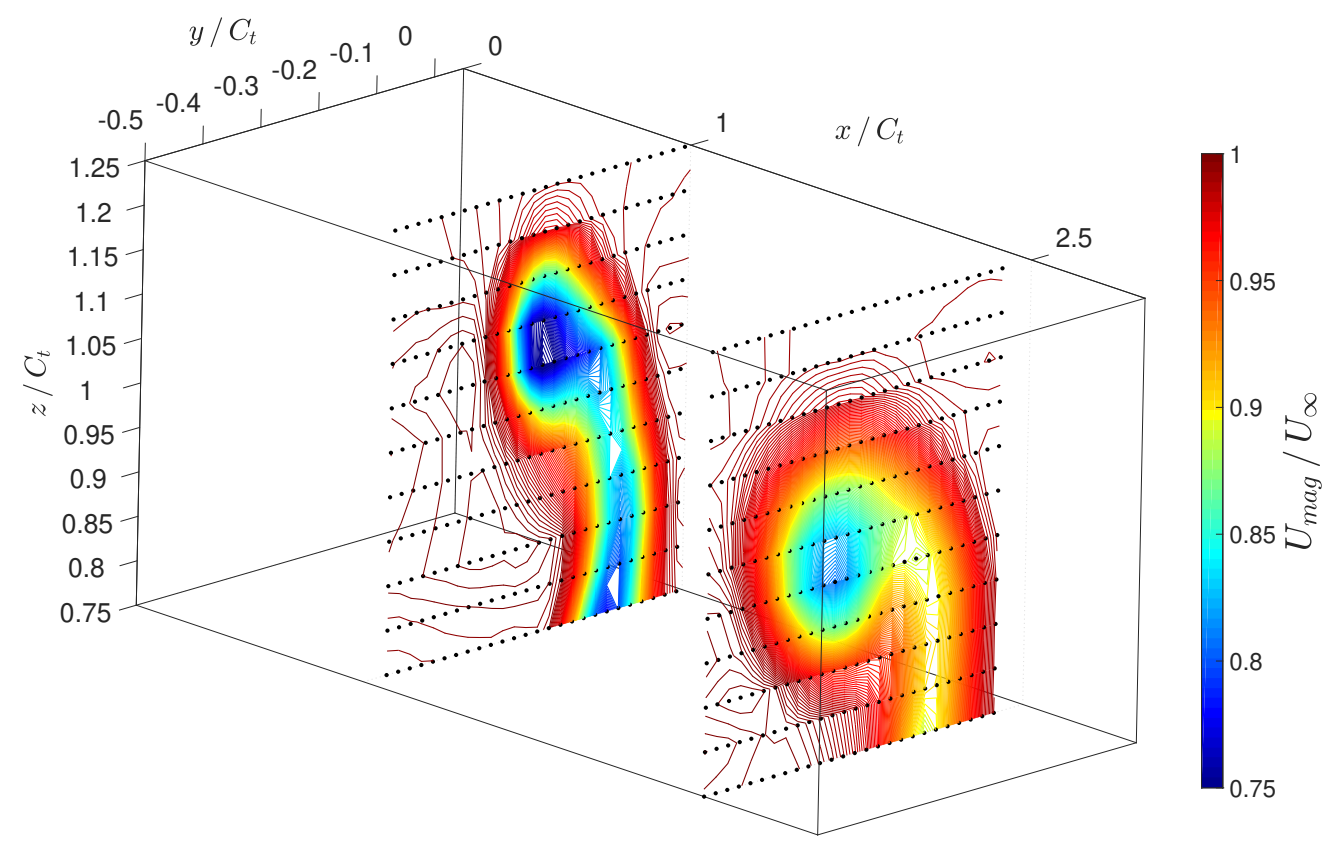

Fig. 6 Contours of $U_{m a g} / U_{\infty}$ for Endplate $E 1_{4}$ shown at $x / C_{t}=1$ and $x / C_{t}=2.5$. Dots indicate the HWA measurement locations.

A $4^{\text {th }}$ order polynomial function was used to calibrate the CTA. Flow velocities between $4.5 \mathrm{~ms}^{-1}$ and $17.25 \mathrm{~ms}^{-1}$ were sampled in steps of $2.125 \mathrm{~ms}^{-1}$ for 30 seconds before and after each spanwise traverse to minimize the influence of temperature drift. The accuracy on the mean flow data obtained using the HWA was estimated to be within $\pm 0.5 \%$, while the turbulence intensity data were estimated to be accurate within $\pm 4 \%$.

HWA data were sampled at two planes, $x / C_{t}=1$ and 2.5, for $E 1_{1}-E 1_{4}$. For the shorter, Group 1 endplates, $E 1_{5}-E 1_{6}$, the measurement planes were located at $x / C_{t}=1$ and 4 . Measurements for the Group 2 endplates were limited to only one streamwise plane, positioned at $x / C_{t}=2$.

The sampling of the tip vortex was performed by taking HWA measurements with a spacing of $2 \mathrm{~mm}$ and $5 \mathrm{~mm}(0.02$ $C_{t}$ and $0.05 C_{t}$ ) in the $y$ and $z$-direction, respectively. Figure 6 shows a graphical representation of the measurement domain for endplate $E 1_{4}$ together with the contours of velocity magnitude $U_{m a g} / U_{\infty}$; the black dots represent the actual 
measurement locations.

Stereo-PIV was conducted for endplates $E 1_{1}$ and $E 1_{4}$. Two cameras as well as the laser and the associated optics were mounted on two parallel traverse systems. After an initial optical calibration, where a calibration target was displaced by $\pm 2 \mathrm{~mm}$ normal to the cameras, the set-up allowed numerous stereo-PIV planes to be acquired. Additionally, the calibration was refined by performing a self-calibration on the particle images [29].

The laser sheet was shone into the test section through a window in the wind tunnel floor. It was aligned in the streamwise direction and allowed measurements from $x / C_{t} \approx 1$ to $x / C_{t} \approx 2.5$. The measurement planes were located between $60 \mathrm{~mm}$ inboard to $5 \mathrm{~mm}$ outboard of the wingtip and were spaced at $5 \mathrm{~mm}$ intervals. A qualitative schematic of the experimental set-up is shown in Figure 7



Fig. 7 Schematic illustration of the stereo-PIV set-up.

A Litron Dual Power $200 \mathrm{Nd}$ :YAG laser with a maximum output energy of $200 \mathrm{~mJ} / \mathrm{pulse}$ and a wavelength of $532 \mu \mathrm{m}$ was used to illuminate the flow field. It was operated in a dual pulse mode and two CCD FlowSense EO 16 Mpix cameras, acquiring at $2 \mathrm{~Hz}$, were positioned on one side of the laser sheet. Schleimpflug mounts were used to achieve a uniform focus across the entire field of view. For each measurement plane, 500 images were acquired to ensure converged statistics. These were dewarped onto the common plane identified by the self-calibration procedure [29]. At the centre of the images, a digital resolution of approximately $14.5 \mathrm{pix} / \mathrm{mm}$ was achieved.

The flow was seeded using Fog fluid nebulised into particles with an average size of $2 \mu \mathrm{m}$.

The time delay between the two exposures was set to $35 \mu \mathrm{s}$, thus resulting in an average particle displacement of 10 pixels. Considering the typical error in determining the peak in the cross-correlation algorithm ( 0.1 pixel), this results 
into an error in the measurement of the mean velocity components of $\pm 1 \%$.

The images were processed using an adaptive algorithm as outlined by Astarita [30] and Giordano \& Astarita [31], with an interrogation window size of 32 pix $\times 32$ pix $(2.20 \mathrm{~mm} \times 2.20 \mathrm{~mm})$ and an overlap of $75 \%$, resulting in a vector pitch of $0.55 \mathrm{~mm}$. To reduce the effect of any undesired reflections within the test section, the historical minimum was removed from each raw image.

\section{Results and Discussion}

In the following section, all the coefficients calculated from force measurements are plotted with their respective uncertainty. The uncertainty in each coefficient is calculated assuming Gaussian uncertainty propagation. The wing area error is assumed negligible compared with the uncertainties in velocity and force readings.

It is further noted, that the velocity magnitude measured using the HW will be referred to as $U_{m a g}$, whilst $U, V$ and $W$ are the velocity components aligned with the $x, y$ and $z$-axis; $u^{\prime}$ refers to the root-mean-square (rms) of the turbulent velocity fluctuations.

\section{A. Effect of the Endplate Tip Geometry Modification}

\section{Aerodynamic Coefficients}

We begin by evaluating the aerodynamic coefficients $C_{l}=L /\left(q_{\infty} S\right)$ and $C_{d}=D /\left(q_{\infty} S\right)$ for the wing equipped with

the Group 1 endplates. Here $q_{\infty}=1 / 2 \rho U_{\infty}^{2}$ is the asymptotic dynamic pressure and $S$ is the wing planform area. Data are plotted as a function of the angle of attack, $\alpha$.

Figures 8 and $8 \mathrm{p}$ show the lift and drag coefficient when the individual Group 1 endplates $\left(E 1_{1}-E 1_{6}\right)$ are mounted to the wing as well as when no endplate is present. As to be expected, $C_{l}$ and $C_{d}$ grow with increasing angle of attack. However, it is difficult to observe any trends caused by the fractal iterations along the top edge of the endplate. The effect of the endplate geometry is highlighted looking at the percentage differences calculated as $\Delta C / C_{r e f}=C_{i}-C_{r e f} / C_{r e f}$, where $C_{i}$ represents the lift (Figure 8d) or drag coefficient (Figure 8p) of the i-th endplate at a given angle of attack and $C_{r e f}$ the value of the coefficient obtained with the clean wing (baseline). While the values of the lift coefficient are found to be quite independent of the multiscale geometry, the drag coefficient shows that the investigated endplates may lead to drag reductions of the order of $10 \%\left(E 1_{6}\right)$. Furthermore, the results suggest that the endplates show an optimal value of the angle of attack at which the drag reduction is the largest. It must be pointed out though that the results presented here are not conclusive in this sense and further investigations with a larger parametric space are needed to conclusively address this point. In addition to this, the large differences outlined in the case of $\alpha=0$ are effectively associated with the fact that, at that particular angle of attack, the accuracy of the system is the lowest.

A more quantitative comparison can be carried out by looking at the aerodynamic efficiency, $C_{l} / C_{d}$, plotted as a function of the angle of attack, as shown in Figure 8 e (and further highlighted by the plot showing the differences 


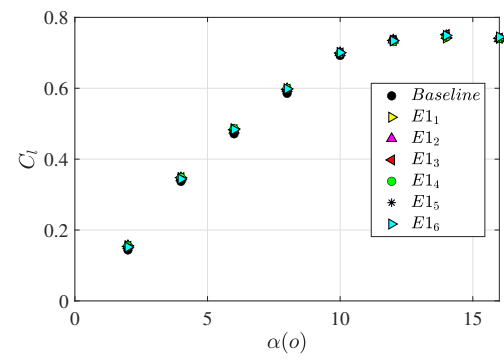

(a)



(d)



(b)

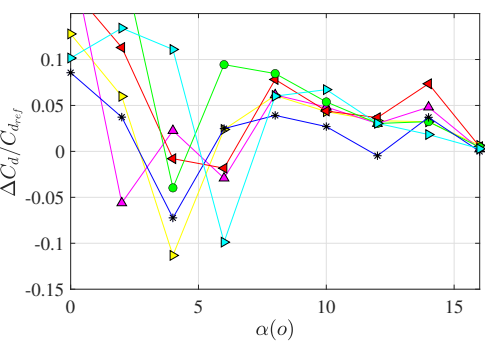

(e)

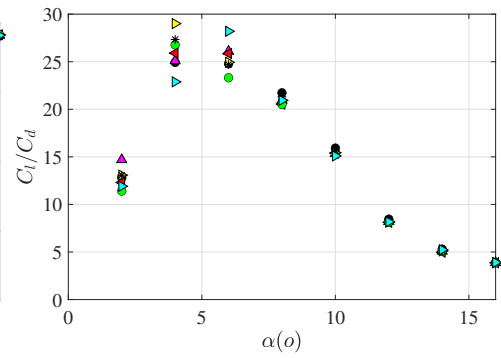

(c)

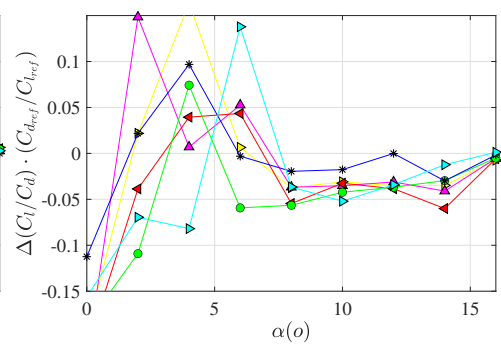

(f)

Fig. 8 Lift coefficient (a), drag coefficient (b) and aerodynamic efficiency (c) as a function of the angle of attack $\alpha$ for the endplates belonging to Group 1. The measurements for the clean wing are also shown for reference. Percentage variation of the lift (d) drag (e) coefficients and aerodynamic efficiency (f) with respect to the reference case (clean wing).

in aerodynamic efficiency, Figure $8 \mathrm{f}$ ). The largest differences can be observed when $2^{\circ}<\alpha<8^{\circ}$. In this range, the efficiency of almost all the endplates is larger than that of the clean configuration when no endplate is mounted to the wing. The highest efficiency is achieved by $E 1_{1}$. When considering only the results from the endplates $E 1_{1}$ to $E 1_{4}$ (characterized by the largest value of $h$ ), it is impossible to identify a clear relationship between performance and the fractal iteration. Therefore, from this analysis no appreciable effect on the aerodynamic forces for the investigated Reynolds numbers can be observed, despite the potential for beneficial downstream effects, which will later be explored. It is also worth explicitly noting that the limited resolution, in terms of angle of attack limits, the capability in determining the exact location of the peak in aerodynamic efficiency. Nevertheless, the obtained trends were deemed sufficient to highlight the potential improvement of the aerodynamic efficiency when a fractal endplate was employed.

Nevertheless, the endplate $E 1_{6}$, which is characterized by a smaller height, $h$, and same amplitude of the fractal modifications, $a$, compared to $E 1_{1}-E 1_{4}$ (see Table 2 ) achieves an efficiency which is very close to $E 1_{1}$. At the same time the efficiency is larger than that obtained by endplate $E 1_{5}$, which features no fractal pattern along its top edge and is of the same height as $E 1_{6}$. The higher efficiency of $E 1_{6}$ with respect to the other fractal geometries may be related to the larger value of $a / h$. This suggests that the fractal geometry does have an effect, but it also indicates that this effect can be reduced when $a / h$ falls below a certain threshold. 


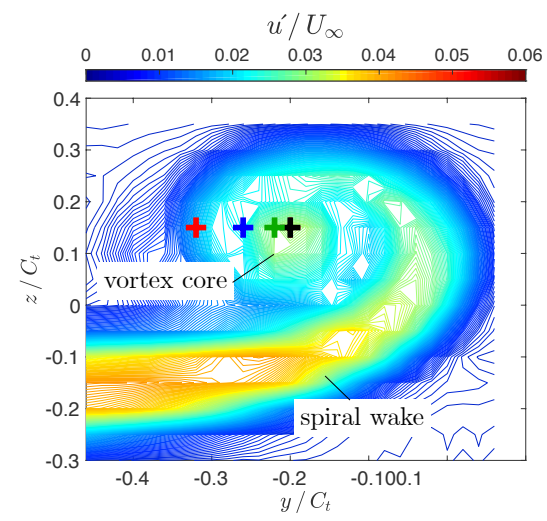

(a)

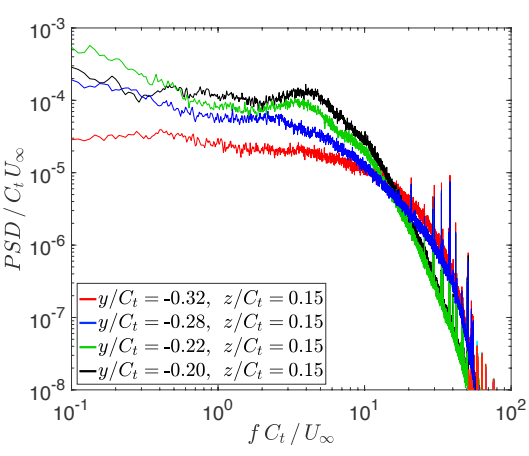

(b)

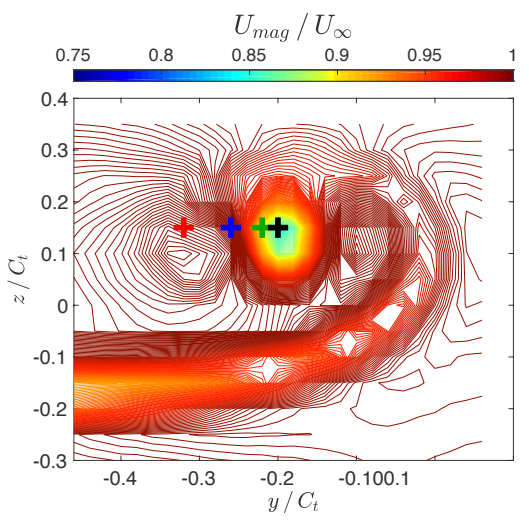

(c)

Fig. 9 Contour representation of the velocity fluctuation $u^{\prime} / U_{\infty}$ (a) and velocity magnitude $U_{m a g} / U_{\infty}$ (c) for the isolated wing. (b) Power spectra at specified locations within the vortex, as represented in the (a) and (c) panels. The color code of the markers corresponds to each individually plotted $P S D$. Data are measured at $x / C_{t}=2.5$. The angle of attack is set to $\alpha=6^{\circ}$.

\section{Near Wake Topology}

HWA measurements allow a comparative analysis of the topology of the wingtip vortex. We begin by observing the flow structure of a clean wing when no endplate is mounted.

Figures $9 \mathrm{p}$ and $9 \mathrm{k}$ show the rms $\left(u^{\prime} / U_{\infty}\right)$ and the velocity magnitude $\left(U_{m a g} / U_{\infty}\right)$, respectively, as measured at $x / C_{t}=2.5$. Here a clear spiral wake, embedding a well defined and confined vortex core can be seen.

The power spectra at various positions inside the wing tip vortex provide further information about its structure and the origin of the turbulent fluctuations. Figure $9 \mathrm{p}$ shows the power spectra at $x / C_{t}=2.5$ for the wing when no endplate is fitted. Each marker on the contour plots is color coded to match the corresponding non-dimensionalised $P S D$ line in Figure $9 \mathrm{p}$.

The energy retained in frequencies below $f C_{t} / U_{\infty}=10$ increases significantly as the vortex core is approached compared to the distribution measured in the spiral wake. Moreover, the energy of small scale turbulence at $f C_{t} / U_{\infty}>20$ drops in the vortex core compared to that in the wake spiral. This agrees well with results presented by Devenport et al. [26], where it was postulated that the results indicate that the core produces very little of its own turbulence. The fluctuating axial energy in the low frequencies was attributed to the buffeting of the vortex core by the spiral wake.

To verify that vortex wandering has not unduly affected the results, the HWA signal is high-pass filtered at at $f C_{t} / U_{\infty}=3$. Low frequency contributions to the measured signal, as caused by vortex wandering, are therefore removed. Whilst the magnitude of $u^{\prime} / U_{\infty}$ is reduced, little difference in the structure of the vortex is observed between the unfiltered and filtered signals shown in figures 10 , thus suggesting that vortex wandering only has a negligible effect on the measured data and the resulting vortex contours. It must be pointed out that the measurements are carried out at a 
relatively small distance downstream of the wing model, hence the effect of the vortex meandering may still be quite limited.

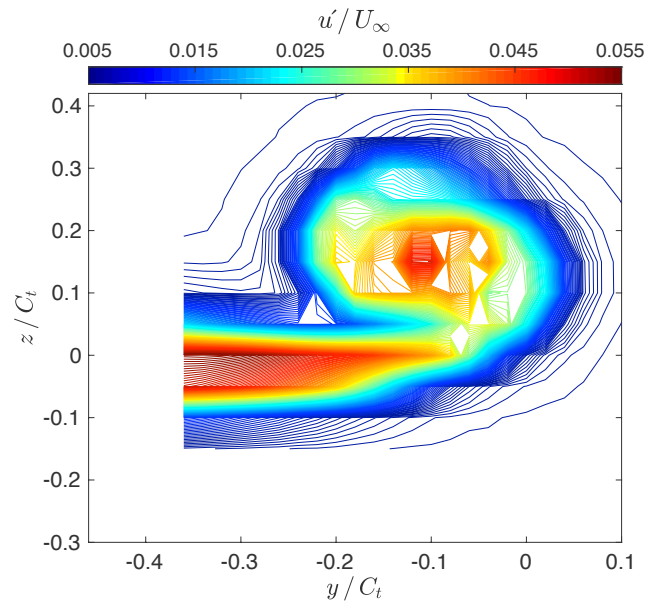

(a)

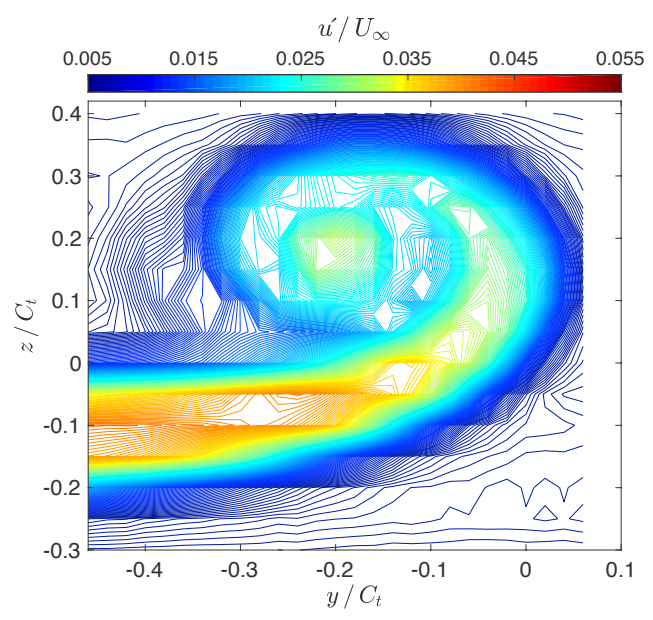

(c)

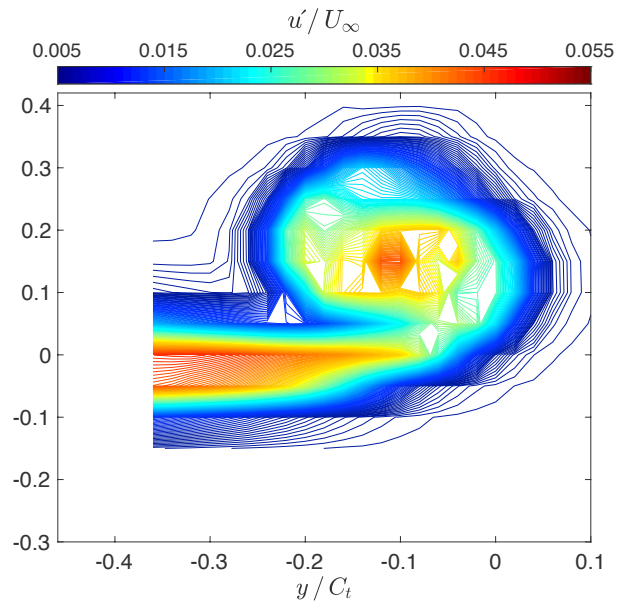

(b)

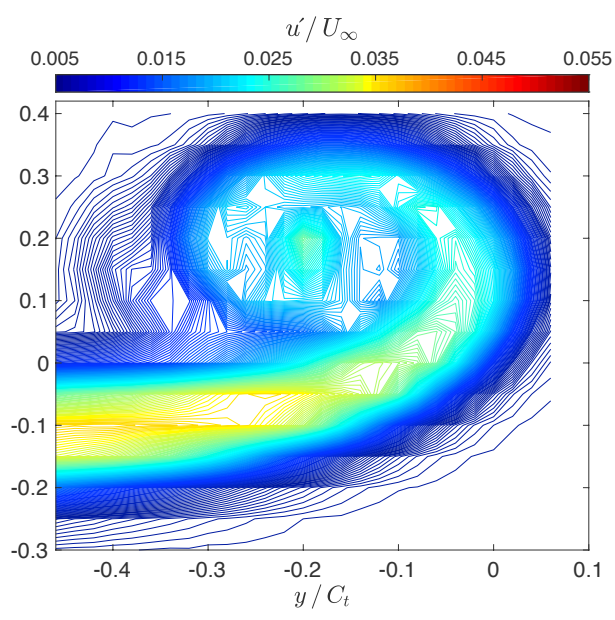

(d)

Fig. 10 Contours of $u^{\prime} / U_{\infty}$ unfiltered a), and high-pass filtered at $f C_{t} / U_{\infty}=3 \mathbf{b}$ ). Data in the first row (a,b) are measured at $x / C_{t}=1$, while data in the second row (c,d) are measured at $x / C_{t}=2$.

The vortices shed by the individual endplates is now analysed using the same methodology.

The first and third columns of Figure 11 , show the contours of $u^{\prime} / U_{\infty}$ and $U_{m a g} / U_{\infty}$, respectively, at $x / C_{t}=2.5$ for $E 1_{1}-E 1_{4}$. Increasing the number of fractal iterations from 0 to 3 , the vortex grows in size whilst featuring a larger velocity deficit and increased levels of turbulence intensity. Furthermore, the vortex becomes more diffused with the distinction between the vortex core and the surrounding vortical fluid becoming less clear.

The second column of Figure 11 shows the power spectra at $x / C_{t}=2.5$ corresponding to the vortex released by each endplate. The measurement locations, for which the power spectra are displayed, are also indicated in the $u^{\prime} / U_{\infty}$ and $U_{m a g} / U_{\infty}$ contour plots. . 
Figure 11p, corresponding to the non-fractal endplate, shows that the PSD levels are almost twice as high at frequencies below $f C_{t} / U_{\infty}=2$ and slightly elevated between $f C_{t} / U_{\infty}=2$ and $f C_{t} / U_{\infty}=10$ inside the vortex core compared to the surrounding wake. Above $f C_{t} / U_{\infty}=20$, the spectral levels in the vortex core drop below what is measured in the wake spiral.

The observations made for the non-fractal endplate also apply to $E 1_{2}$, Figure 11 , with the difference between the energy in the high frequency fluctuations inside and outside the center of the vortex being less pronounced.

For $E 1_{3}$ and $E 1_{4}$, Figures 11 and 11 k, the power spectra is found to collapse for most locations inside the vortex, suggesting a uniformly distributed energy level throughout the entire vortex.

The results presented in regards to the power spectra at various locations inside the vortex lead to the conclusion that the vortex becomes more diffused with increasing fractal iteration. This was previously hypothesised based on the vortex contours of $u^{\prime} / U_{\infty}$ and $U_{m a g} / U_{\infty}$ and has been further confirmed, since no marked difference can be detected in the power spectra between the centre of the vortex and its surrounding for the higher fractal iterations. The power spectra at different locations, unlike for the plain wing and the non-fractal endplate, collapse with a clear distinction between vortex core and surrounding wake, as shown in Figure 11p, no longer being present.

Scanning stereo-PIV results can be particularly helpful in providing a clear and comprehensive overview of the wingtip vortex topology in the two limiting cases of $E 1_{1}$ and $E 1_{4}$. The three dimensional visualization of the flow field is obtained by interpolating the measured planes in the spanwise direction. The vortex is subsequently identified using the Q-criterion [32], with $Q=0.018 \mathrm{~s}^{-2}$. The iso-surfaces are color coded using values of $V$. Figure 12 shows the comparison between $E 1_{1}$ and $E 1_{4}$. Two vortical structures can be seen, where the bottom one does not seem to be affected by the change in endplate geometry, whilst the top vortex shows a marked difference. This top vortex shed by $E 1_{1}$, when no fractal pattern is applied to the top edge, is more coherent and less distorted than its counterpart produced by the fractal endplate.

More quantitative information can be obtained by looking at the normalized maximum transverse velocity around the vortex, $\left(U_{t_{\max }}\right)_{a v} / U_{\infty}$. This is calculated by creating a plane perpendicular to the oncoming flow at each streamwise $x$-location. The maximum transverse velocity, measured at each streamwise PIV plane along the upper and lower half of the wingtip vortex located on this perpendicular plane, is now found as shown in Figure 13 and the result averaged,

$$
\left(U_{t_{\max }}\right)_{a v}=\frac{1}{N} \sum_{k=1}^{N}\left(\sqrt{V^{2}+W^{2}}\right)_{k, \max }
$$

where $\mathrm{N}$ is the number of measurement points and $\left(\sqrt{V^{2}+W^{2}}\right)_{k, \max }$ is the maximum transverse velocity at each location, $k$, along the outside of the vortex.

As shown in Figure 14 , the vortex released by Endplate $E 1_{4}$ shows a reduction of $\left(U_{t_{\max }}\right)_{a v} / U_{\infty}$ by $30 \%$ compared to the vortex shed by Endplate $E 1_{1}$. This reduction in tangential velocity helps to reduce the rolling hazard for aircraft 




(a) $E 1_{1}$

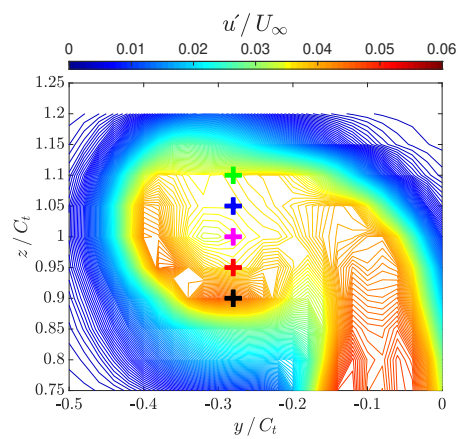

(d) $E 1_{2}$

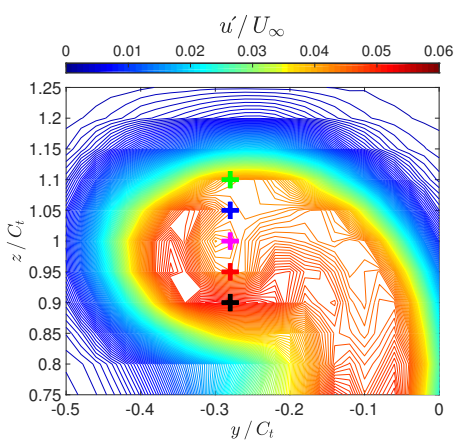

(g) $E 1_{3}$



(j) $E 1_{4}$

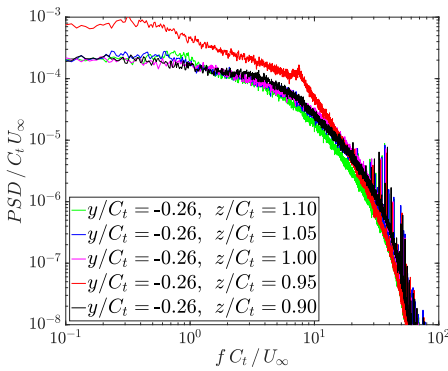

(b) $E 1_{1}$

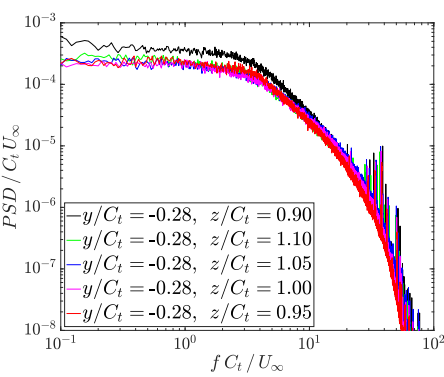

(e) $E 1_{2}$

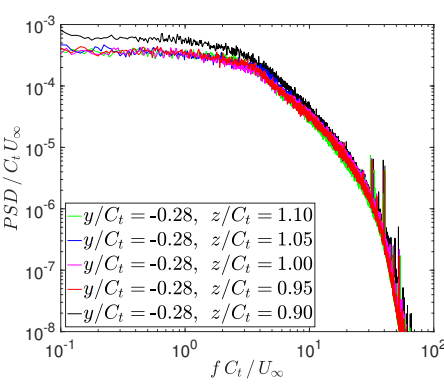

(h) $E 1_{3}$

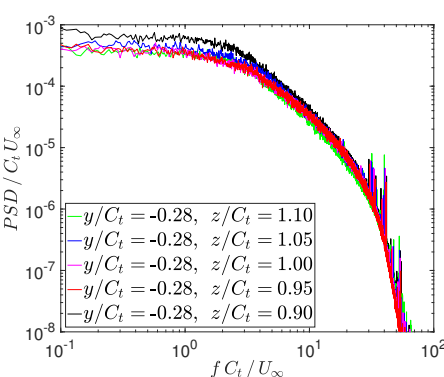

(k) $E 1_{4}$

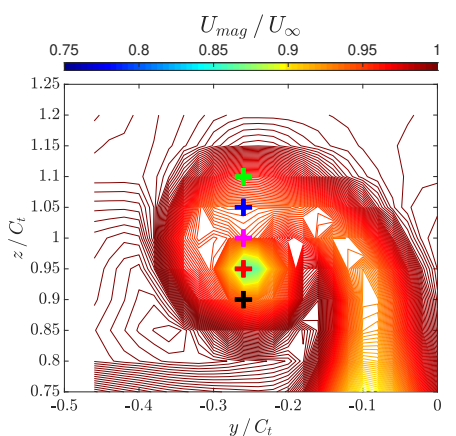

(c) $E 1_{1}$



(f) $E 1_{2}$



(i) $E 1_{3}$



(1) $E 1_{4}$

Fig. 11 Contours of $u^{\prime} / U_{\infty}$ (a), (d), (g), (j), power spectra at indicated locations (b), (e), (h), (k) and $U_{m a g} / U_{\infty}$ (c), (f), (i), (l) at $x / C_{t}=2.5$. The markers are color coded according to each individually plotted $P S D$. Data are measured at $x / C_{t}=2.5$. The angle of attack is set to $\alpha=6^{\circ}$. 




(a) $E 1_{1}$

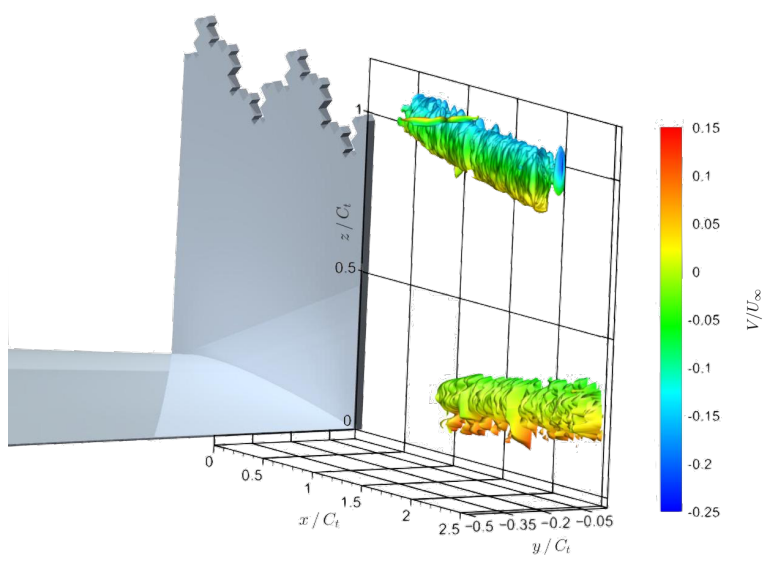

(b) $E 1_{4}$

Fig. 12 Iso-surfaces of $Q /\left(U_{\infty}^{2} / c_{t}^{2}\right)=\mathbf{0 . 0 1 8}$ for Endplates $E 1_{1}$ and $E 1_{4}$, colored by contours of $V$. The angle of attack is set to $\alpha=6^{\circ}$.



(a) $E 1_{1}$

Fig. 13 Schematic illustration of how $\left(U_{t_{\max }}\right)_{a v} / U_{\infty}$ is calculated. 
encountering the vortex wake as well as to reduce the induced drag experienced by the wing.

A characteristic vortex dimension can be defined as $r=\frac{z_{V_{\max }}-z_{V_{\min }}}{2}$, where $z_{V_{\max }}$ and $z_{V_{\min }}$ are the vertical locations of the maximum and minimum $V$. As shown in Figure 14 p the vortex created by Endplate $E 1_{4}$ is larger and grows more quickly compared to the vortex shed by Endplate $E 1_{1}$. Even though $\left(U_{t_{\max }}\right)_{a v} / U_{\infty}$ remains almost invariant throughout the measurement domain, the faster growth rate suggests that an earlier decay of the wingtip vortex shed by $E 1_{4}$ is possible, as higher rates of diffusion occur at the vortex edge.

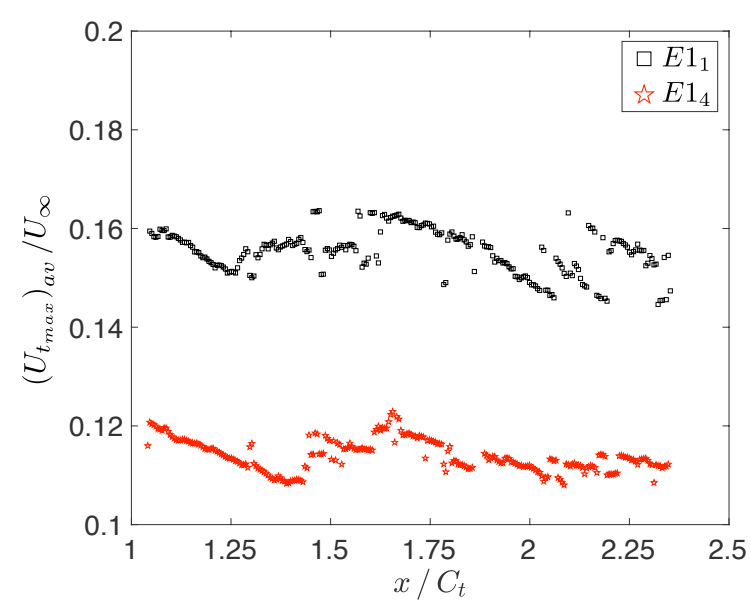

(a)

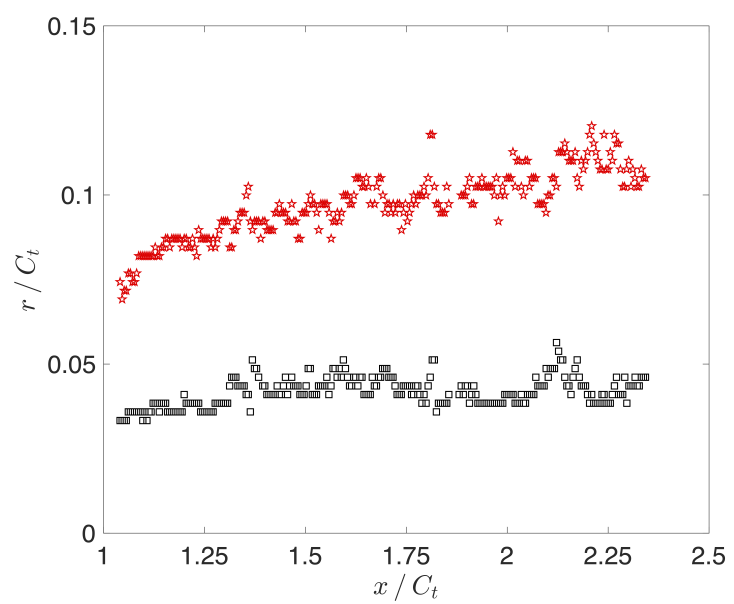

(b)

Fig. $14 U_{t_{a v}} / U_{\infty}$ (a), and $r / C_{t}=\frac{z v_{\max }-z v_{\min }}{2 C_{t}}$ (b) for Endplates $E 1_{1}$ and $E 1_{4}$.

Evidence for varying levels of energy at different associated frequencies within the wingtip vortex was presented in the preceding section. Since the HW data was sampled at $50 \mathrm{kHz}$, the data can be high-pass filtered in order to distinguish the individual sources of velocity fluctuations. Contours plots of $u^{\prime} / U_{\infty}$ for the unfiltered signal are presented in the top row of Figure 15. The bottom row shows the results when the signal is high-pass filtered at $f C_{t} / U_{\infty}=40$ for Endplates $E 1_{1}-E 1_{4}$ at $x / C_{t}=2.5$.

The vortex core for the non-fractal endplate is no longer clearly identifiable once the signal is high-pass filtered at $f C_{t} / U_{\infty}=40$, as shown in Figure 15 . This suggests that the high turbulence intensity recorded in the vortex core is due to large scale coherent structures.

Conversely, even though the filtering operation leads to a lower value of the turbulence intensity level, the filtered and unfiltered contour plots obtained for the fractal endplates do not significantly change in shape.

It is worth noting that the regions of high turbulence intensity in the inboard section of the vortex $\left(y / C_{t} \approx-0.4\right.$, Fig. $15 \mathrm{p}$ and c) are also no longer clearly identifiable when the signal has been high-pass filtered. This shows, as it was observed for the non-fractal endplate, that the smallest velocity fluctuations are not in fact large contributors to the unfiltered high turbulence intensity measured at these locations. 


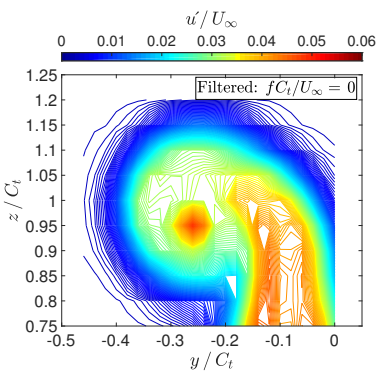

(a) $E 1_{1}, f C_{t} / U_{\infty}=0$.



(e) $E 1_{1}, f C_{t} / U_{\infty}=40$.

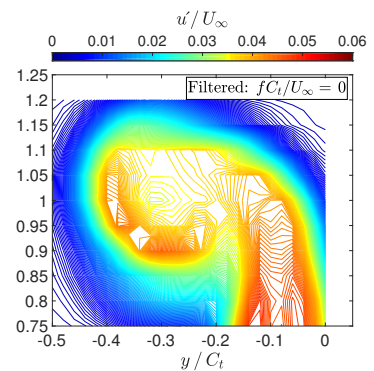

(b) $E 1_{2}, f C_{t} / U_{\infty}=0$.

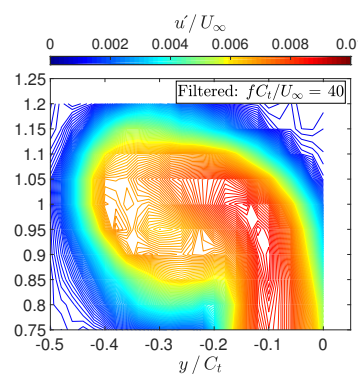

(f) $E 1_{2}, f C_{t} / U_{\infty}=40$.

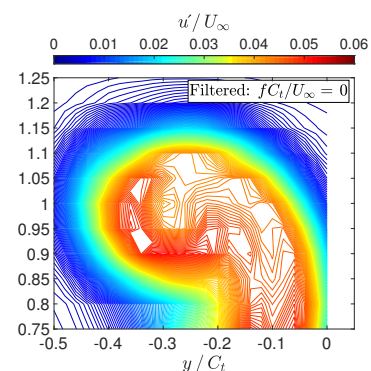

(c) $E 1_{3}, f C_{t} / U_{\infty}=0$.

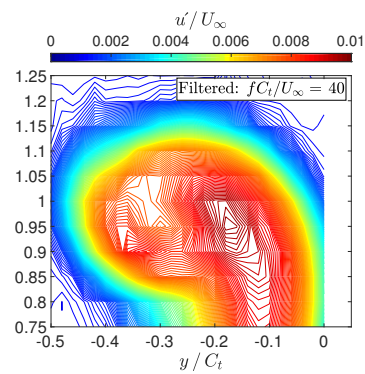

(g) $E 1_{3}, f C_{t} / U_{\infty}=40$.

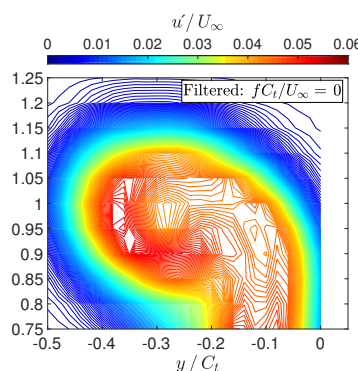

(d) $E 1_{4}, f C_{t} / U_{\infty}=0$.

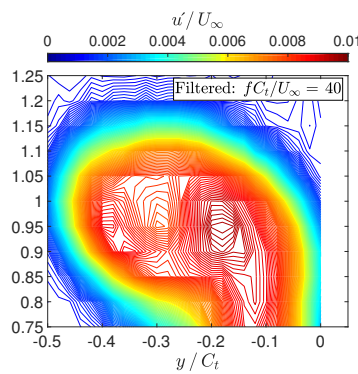

(h) $E 1_{4}, f C_{t} / U_{\infty}=40$.

Fig. 15 Contours of $u^{\prime} / U_{\infty}$ unfiltered a) - d), and high-pass filtered at $f C_{t} / U_{\infty}=40 \mathbf{e}$ ) - h) at $x / C_{t}=2.5$. The angle of attack is set to $\alpha=6^{\circ}$.

To further investigate this aspect, we look at the vortex topology closer to the wing, at $x / C_{t}=1$, for the endplates $E 1_{1}$ and $E 1_{4}$. Figure 16 shows the $P S D$ as a function of the Strouhal number measured at various location across the vortex for the two cases. The vortex created by the non-fractal endplate features two strong peaks at $f C_{t} / U_{\infty}=6.9$ and at $f C_{t} / U_{\infty}=8.0$. The former is measured in the region corresponding to the wake of the endplate and the latter is measured at the vortex core. In contrast to this, no spikes are observed in the spectrum for $E 1_{4}$. This once more suggests that the vortex is a less coherent and that there thus exists the possibility for it to dissipate more quickly.

The spike in the spectrum at low frequencies is observed for each endplate in the unrolled-up portion of the wake (albeit less evident in the case of $E 1_{2}-E 1_{4}$ ). This indicates that the spike is not created by the vortex itself. Instead it is proposed that the spike at $f C_{t} / U_{\infty}=6.9$ is created by a shedding process similar to that observed behind a rectangular plate, where the Strouhal number based on the freestream velocity and the endplate thickness is 0.206 . As the vortex rolls up, it draws in the endplate wake and thus causes the spike to be measured within the vortex itself as well.

A similar peak was observed in the transverse velocity powerpectra at the vortex core by Terashima et al. [33] at $f C_{t} / U_{\infty}=14, x / C_{t}=0.1$, and $R e=124000$ in an experimental study of a NACA 0012 half span wing with no endplate fitted. The authors attributed the origin of the spike in the powerpectra to the wake of the wing trailing edge and hence come to the same conclusion; albeit with the wing trailing edge rather than the endplate trailing edge causing the observed spike.

A similar flow field analysis is performed for endplates $E 1_{5}$ and $E 1_{6}$, which are characterized by a smaller height $h$ 


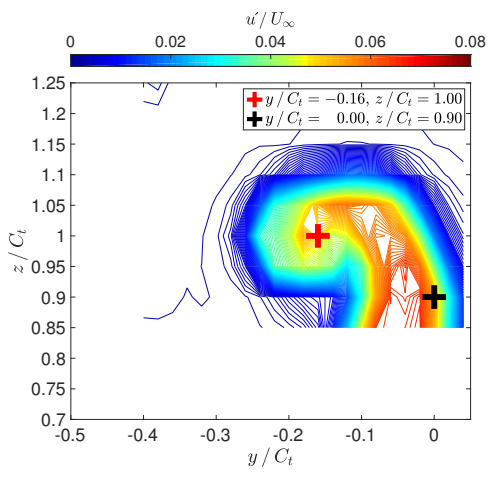

(a) $E 1_{1}$

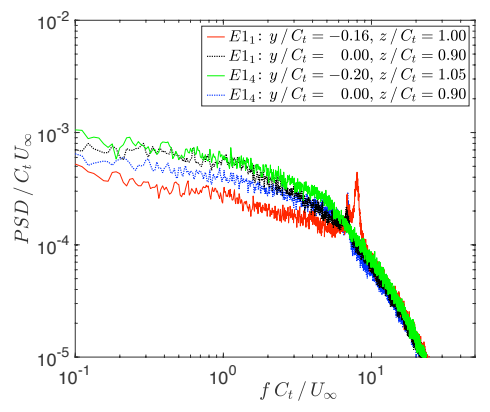

(b) $P S D$ at indicated points

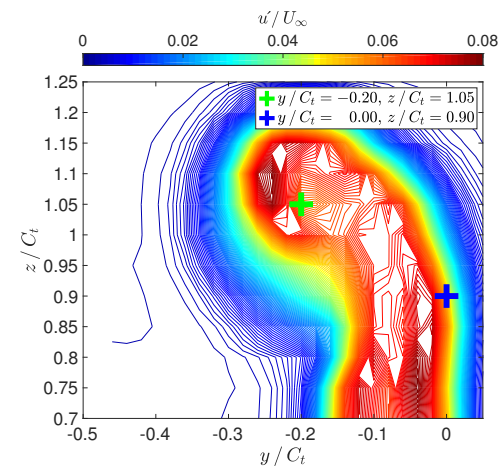

(c) $E 1_{4}$

Fig. $16 P S D$ at $x / C_{t}=1$. The measurement positions inside the vortex and in the unrolled-up portion of the wake for Endplates $E 1_{1}$ and $E 1_{4}$ are color coded to match the displayed spectral plots. Data are measured at $x / C_{t}=2.5$. The angle of attack is set to $\alpha=6^{\circ}$.

(see Table 2). Data are measured at two different streamwise locations, namely $x / C_{t}=1$ and $x / C_{t}=4$.

The contour plots of $U_{m a g} / U_{\infty}$ and $u^{\prime} / U_{\infty}$ and the high-pass filtered $u^{\prime} / U_{\infty}$ in Figure 17 are obtained at $x / C_{t}=1$. Data show that from the vortex topology standpoint, similar conclusions can be drawn regardless the value of $h$. However, at lower values of $a / h$ the fractal geometry causes an upward displacement of the vortex core.

The fractal endplate shows a stronger velocity deficit alongside a more turbulent vortex, compared to the non fractal case, which seems to be still in the earlier stages of formation. Furthermore, the high-pass filter shows little effect on the rms values for $E 1_{6}$. Conversely, the non fractal endplate $E 1_{5}$ shows the disappearance of the well defined vortex core, with more uniform values of turbulence intensity residing within the vortex, once the high-pass filter is applied.

At larger streamwise distances, Figure 18, the non fractal endplate exhibits a strong and well defined vortex core. The fractal endplate $E 1_{6}$ features a much broader vortex core, characterized by large values of turbulence intensity. Similarly to the $E 1_{1}-E 1_{4}$ cases, the peak in turbulence intensity is related to large scales of the flow, as evident by the high-pass filtered contour plot of Figure 18 .

\section{B. Effect of the Outboard Surface Endplate Modification}

We now turn our attention to the Group 2 endplates, which are characterized by a geometric modification to the outboard surface. A similar analysis as applied to the Group 1 endplates is performed. We first examine the aerodynamic coefficients obtained by mounting the individual endplates to the wing. Subsequently, we investigate the vortex produced by the endplate yielding the highest $C_{l} / C_{d}$ and compare it to the clean endplate. All hot wire experiments for Group 2 are carried out at $\alpha=4^{\circ}$. 


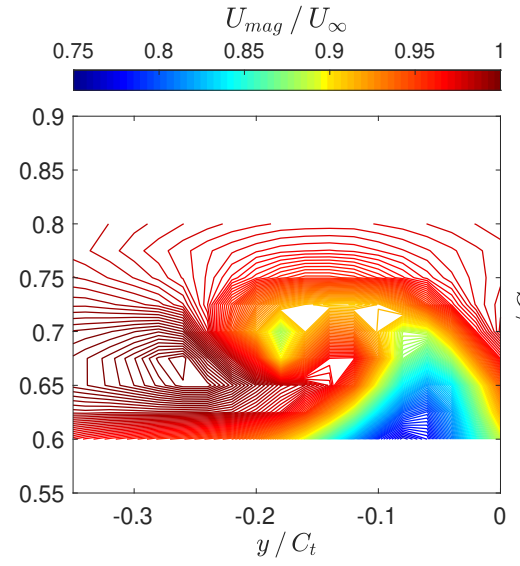

(a) $E 1_{5}$

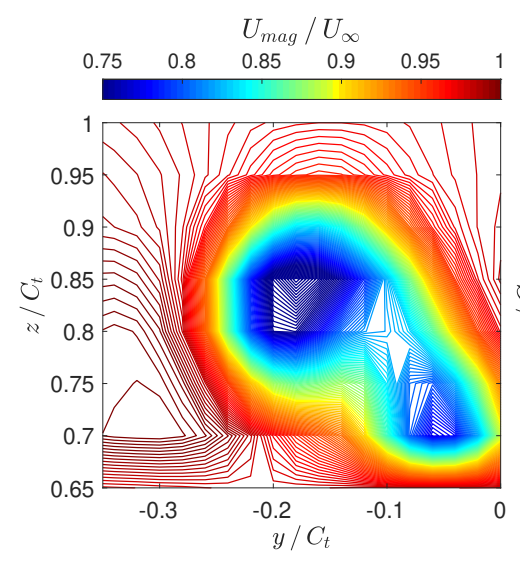

(d) $E 1_{6}$



(b) $E 1_{5}$

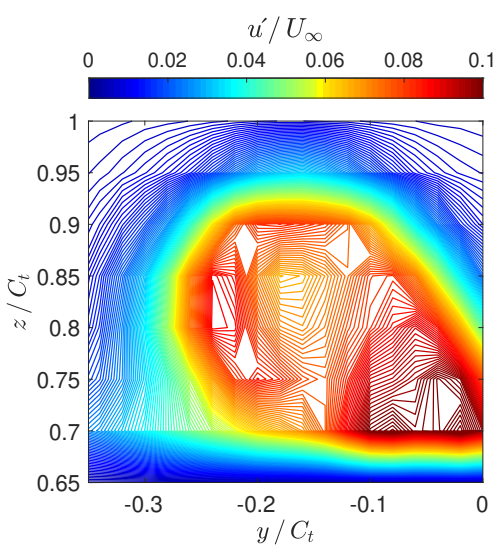

(e) $E 1_{6}$



(c) $E 1_{5}$

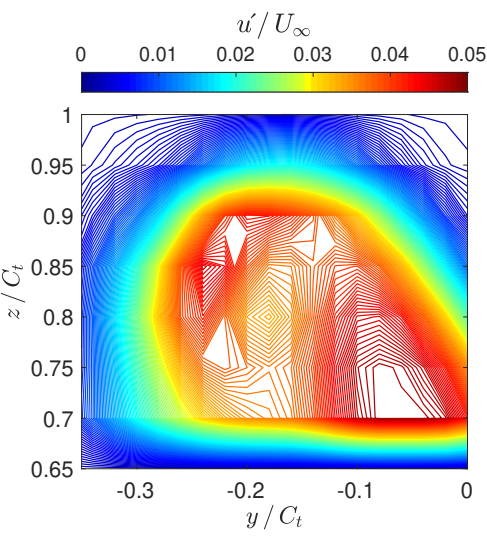

(f) $E 1_{6}$

Fig. 17 Contours of $U_{m a g} / U_{\infty}$ a), d), $u^{\prime} / U_{\infty}$ b), e), and high-pass filtered $u^{\prime} / U_{\infty}$ at $f C_{t} / U_{\infty}=40$ c), f), at $x / C_{t}=1$. 




(a) $\mathrm{E} 1_{5}$

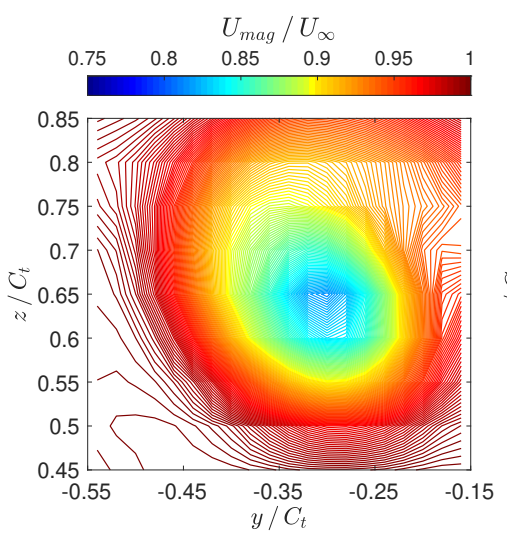

(d) $E 1_{6}$

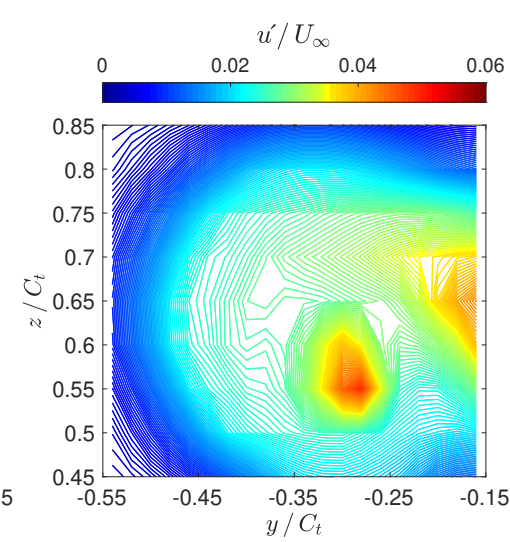

(b) $E 1_{5}$

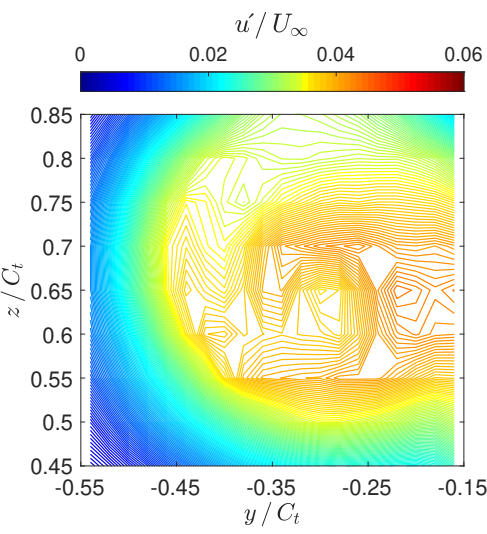

(e) $E 1_{6}$

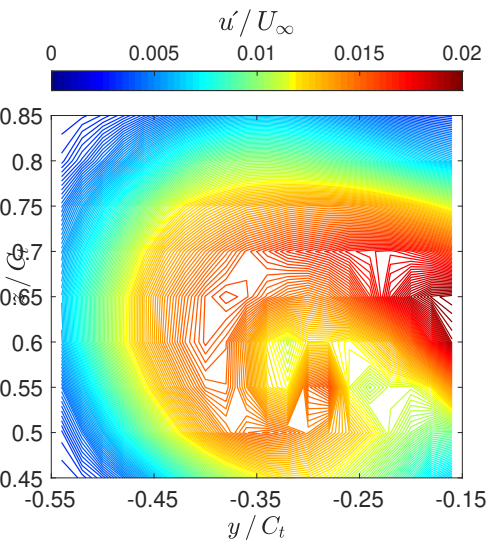

(c) $E 1_{5}$



(f) $E 1_{6}$

Fig. 18 Contours of $U_{m a g} / U_{\infty}(\mathbf{a}),(\mathbf{d}), u^{\prime} / U_{\infty}(\mathbf{b})$, (e), and high-pass filtered $u^{\prime} / U_{\infty}$ at $f C_{t} / U_{\infty}=40(\mathbf{c})$, (f), at $x / C_{t}=4$. 


\section{Aerodynamic coefficients}

Figures $19 \mathrm{l}$-c show the aerodynamic coefficients $C_{l}, C_{d}$ and $C_{l} / C_{d}$ as a function of the angle of attack obtained for the endplates $E 2_{1}-E 2_{4}$. To further highlight the differences associated with the winglet geometry, the percentage difference of each coefficient with respect to the reference case $\left(\Delta C / C_{r e f}\right)$, which in the case of group 2 is the $E 2_{1}$ endplate, were plotted in Figures $19 \mathrm{~d}-\mathrm{f}$.

Similarly to the Group 1 endplates, it is not possible to observe any significant differences from the lift coefficient alone. This was also confirmed when looking at the percentage differences, with nearly all cases showing no influence of the endplate geometry. All endplates cause a similar behaviour of $C_{l}$, which also reflects the same trend observed in Figure $8 \mathrm{a}$ and $\mathrm{d}$ for the clean wing when no endplate is present.

The analysis of the drag coefficient, Figure $19 \mathrm{p}$ and e, reveals some difference between the endplates. In particular, endplate $E 2_{3}$ causes a consistently higher drag, throughout the entire range of investigated angles of attack. The largest differences occur in the range $4^{\circ}<\alpha<10^{\circ}$. As it will be shown later, this aspect is related to the contribution of the small scales introduced by the multiscale geometry, which enhance the viscous effects.

Figure 19: shows the aerodynamic efficiency $C_{l} / C_{d}$ for the four endplates. Consistent with the larger values of $C_{d}$, $E 2_{3}$ exhibits the lowest efficiency. Furthermore, both $E 2_{2}$ and $E 2_{4}$ outperform the baseline endplate, with peaks of $11.1 \%$ and $8.9 \%$ improvement in aerodynamic efficiency, respectively (Figure 19 ).

The two endplates $E 2_{2}$ and $E 2_{4}$ have a similar $C_{l} / C_{d}$ for all the investigated angles of attack. Interestingly, although they feature the same multi-scale pattern (see Table 3) they have a significantly different modified surface area. Whilst for $E 2_{2}$ the entire outboard surface is modified, the multi-scale pattern is only applied to the top portion for $E 2_{4}$ and covers $7.5 \%$ of the surface. This suggests that there is an optimum endplate area that should be modified to improve the aerodynamic efficiency. Manipulating the endplate surface beyond this level may be ineffective or even detrimental.

Figure 20a-b show the spatial distribution of the velocity magnitude in the two investigated cases, $E 2_{1}$ and $E 2_{2}$. The two endplates attain similar values of the velocity deficit. However, the plain endplate shows a significantly larger low velocity region, with the wake of the endplate merging with the tip vortex. On the other hand, the multi-scale endplate features two separate, identifiable, low velocity regions, with the tip vortex clearly separated from the endplate's wake.

Further insights can be gained by looking at the spatial distribution of the axial velocity fluctuations $u^{\prime} / U_{\infty}$, as shown in Figures 20k-d. The plain endplate features higher turbulence intensity levels as well as a broader turbulent region. Similarly to the case of the endplates belonging to Group 1, a high pass filtering of the signal at a normalised frequency $f C_{t} / U_{\infty}=40$ is performed. The results are reported in Figures 20-f. The contours suggest that the tip vortex produced by the $E 2_{1}$ endplate is characterized by a significantly larger energy content at frequencies below the cutoff value. As for the Group 1 endplates, the effect of the multi-scale geometry is such that the tip vortex is characterized by small scale turbulence, thus possibly making it more prone to dissipate.

Furthermore, the normalised PSD at the vortex core at $x / C_{t}=1$ for the clean, $E 2_{1}$, and the multi-scale endplate, 


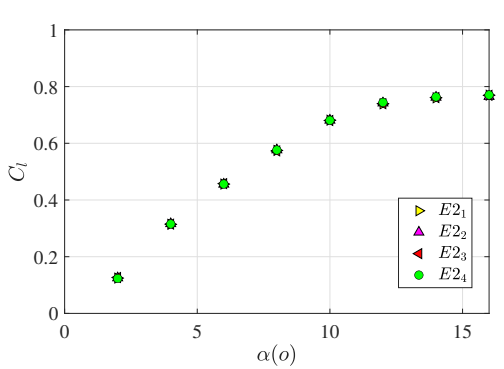

(a)

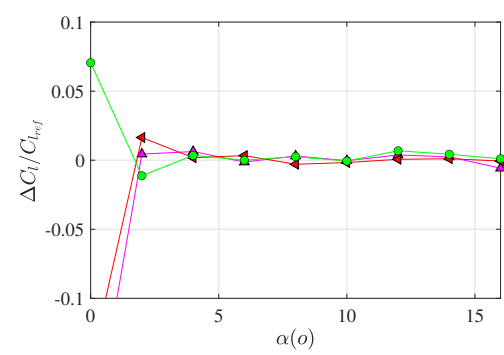

(d)

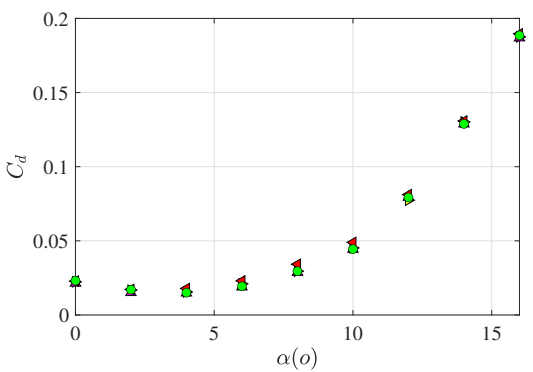

(b)



(e)

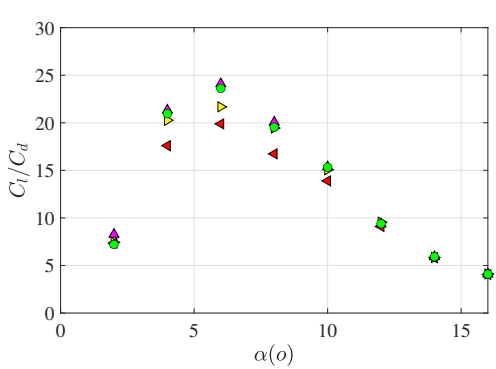

(c)

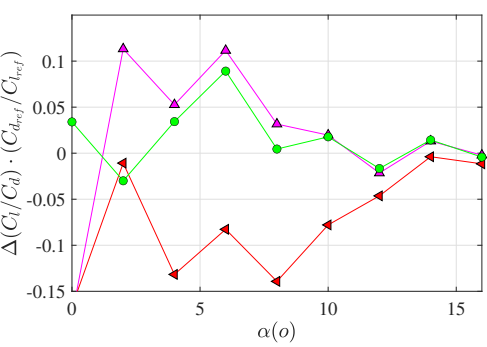

(f)

Fig. 19 Lift coefficient (a), drag coefficient (b) and aerodynamic efficiency (c) as a function of the angle of attack $\alpha$. Data are taken for the Group 2 endplates. Percentage variation of the lift (d) drag (e) coefficients and aerodynamic efficiency (f) with respect to the reference case $\left(E 2_{1}\right)$.

$E 2_{2}$, can be identified and are shown in Figure 21. In the range of normalised frequencies $f C_{t} / U_{\infty}<4$ the spectral energy values obtained for the $E 2_{1}$ are systematically larger than for the multi-scale endplate. This is in line with the previous qualitative observations, that the energy content in the lower frequencies is higher for $E 2_{1}$. Little difference between both endplates can instead be detected for values of $f C_{t} / U_{\infty}>4$. By computing the integral of the PSD for values of $f C_{t} / U_{\infty}<4$ it is found that the $E 2_{2}$ is characterized by energy values $18 \%$ smaller than the baseline configuration. Part of this energy is actually redistributed towards the higher end of the spectrum: when comparing the integral of the PSD for values of the normalised frequency $f C_{t} / U_{\infty}>4.5, E 2_{2}$ features energy values $4 \%$ larger than the $E 2_{1}$.

\section{Conclusions}

In this paper we propose two geometry modifications to a wing endplate in order to mitigate the wingtip vortex. We show that, under certain circumstances, the introduction of multi-scale geometries can be beneficial.

The first geometric modification is achieved by applying an increasingly larger number of fractal iterations to the top edge of the endplate. The results suggest that the fractal endplate generates a wider and more turbulent vortex. However, the higher turbulence intensity is associated with small scale structures, as shown by high pass filtering the velocity signal at a normalised frequency of $f C_{t} / U_{\infty}=40$. The data also suggest that the effectiveness of the fractal endplate is related to the ratio between the amplitude of the modification to the endplates' height, thus suggesting the possible 




(a) Endplate $E 2_{1}, f C_{t} / U_{\infty}=0$.

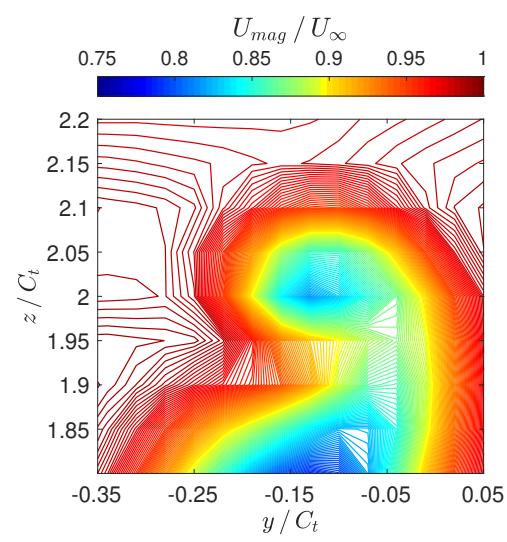

(d) Endplate $E 2_{2}, f C_{t} / U_{\infty}=0$.

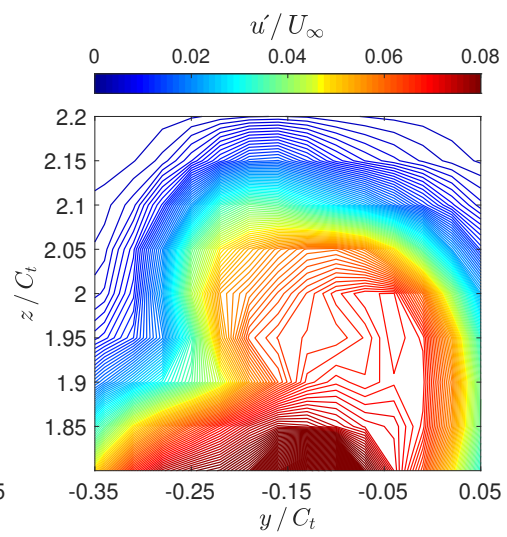

(b) Endplate $E 2_{1}, f C_{t} / U_{\infty}=0$.

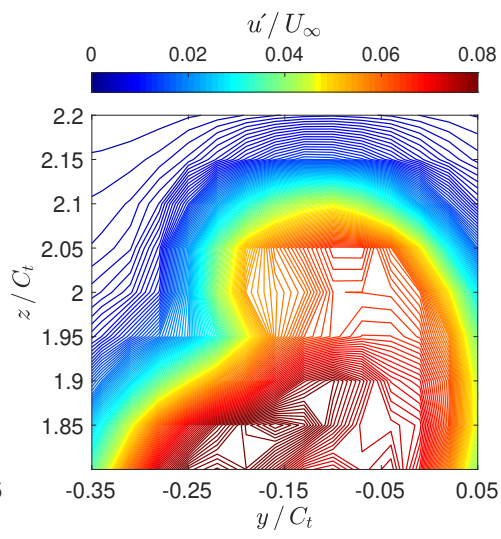

(e) Endplate $E 2_{2}, f C_{t} / U_{\infty}=0$.

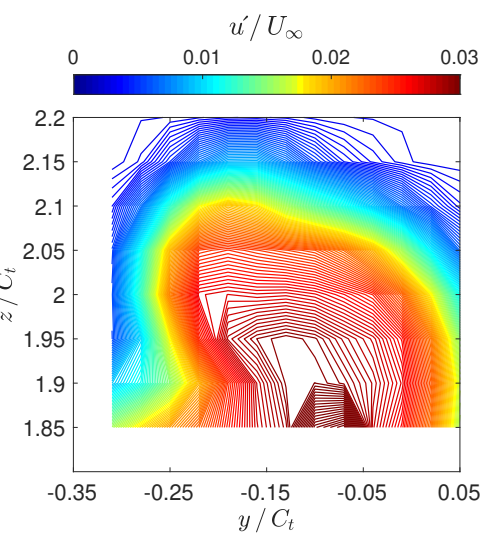

(c) Endplate $E 2_{1}, f C_{t} / U_{\infty}=40$.

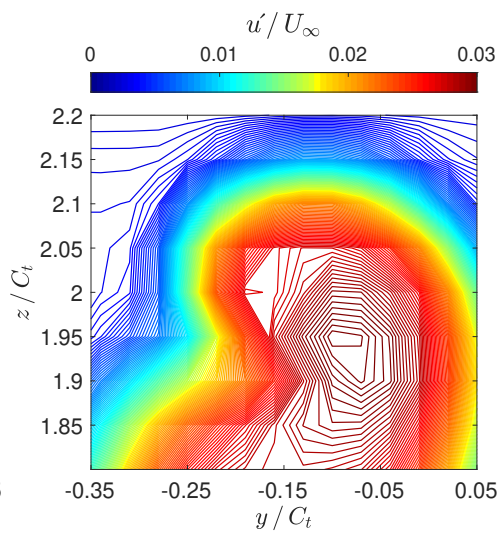

(f) Endplate $E 2_{2}, f C_{t} / U_{\infty}=40$.

Fig. 20 Contours of $U_{m a g} / U_{\infty}$ a), d), $u^{\prime} / U_{\infty}$ b), e), and high-pass filtered $u^{\prime} / U_{\infty}$ at $f C_{t} / U_{\infty}=40$ c), f), at $x / C_{t}=2$. 


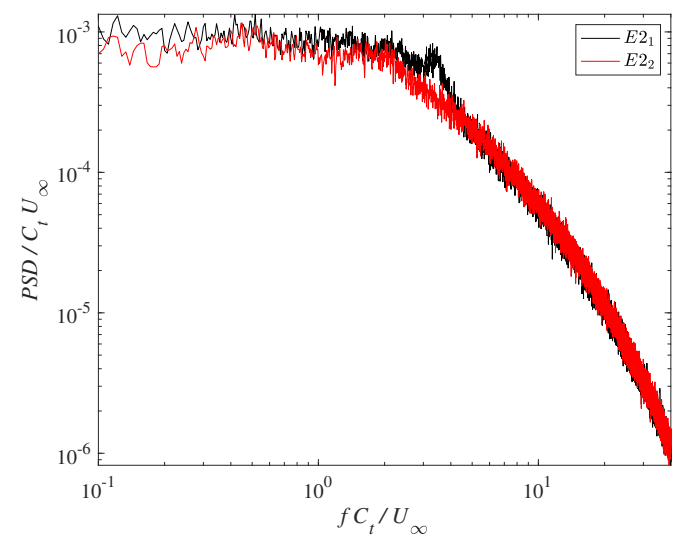

Fig. 21 Power spectra for Endplate $E 2_{1}$ and Endplate $E 2_{2}$ at $x / C_{t}=2$ at the vortex core.

existence of an optimum geometry. Nevertheless, a more extensive data-set is needed to support this conclusion.

The second geometric modification is the application of a multi-scale pattern to the outboard surface of the endplate. The results clearly suggest that depending on the choice of the characteristic lengthscales $\left(l_{0}\right.$ and $\left.l_{1}\right)$ and of the size of the manipulated surface area $\left(S_{m}\right)$, a beneficial or detrimental effect can occur. Particularly relevant is the fact that two endplates characterized by significantly different $S_{m}(7.5 \%$ and $100 \%)$ and same $l_{0}$ and $l_{1}$ lead to similar values of the aerodynamic efficiency $C_{l} / C_{d}$. This suggests that introducing a manipulation on the whole outboard facing surface might be detrimental and that $S_{m}$ should be carefully optimized when designing the multi-scale endplate.

Even though the nature of the geometric manipulation is different for the two groups of endplates, the result obtained by introducing a multi-scale manipulation is quite similar. Regardless of its location, the multi-scale modification drains energy from the large structure $(\approx 18 \%)$ and shifts this energy towards the small scale eddies $(\approx 4 \%)$. Consistently, the wingtip vortex produced by a multi-scale endplate is also more uniform, as a result of the inherent isotropy of the flow at the smaller scales.

The study was intended as a conceptual investigation into the effect of multi-scale geometries on the wingtip vortex. The hope is that these first initial results can stimulate further research investigating the far-field vortex development as well as compressibility effects occurring at the cruise velocities of commercial aircraft. A further evaluation is especially important as the introduction of multi-scale geometries, along the top edge or the outboard surface of the endplate, appears to contrast the development direction of aircraft manufacturers, where a clean winglet design is prioritised. However, this case study is seen as the possibility of introducing ad hoc manipulations to the winglet geometry when needed, thus allowing for the actuation of a portion of the outward facing surface of the endplate tips during take-off and landing and a laminar winglet during cruise. Moreover, an investigation exploring the relationship between Reynolds number and the optimal number of scales would also be of interest. An actual implementation of a multi-scale pattern would need a careful optimization process for the particular application. 


\section{Acknowledgements}

The authors were supported by the ERC Advanced grant (Grant agreement ID: 320560) awarded to JCV. GC acknowledges Prof. Tommaso Astarita for providing the code to perform the PIV processing.

\section{References}

[1] Crow, S. C., "Stability theory for a pair of trailing vortices," AIAA Journal, Vol. 8, No. 12, 1970, pp. $2172-2179$. https://doi.org/10.2514/3.6083.

[2] Widnall, S. E., Bliss, D., and Zalay, A., "Theoretical and Experimental Study of the Stability of a Vortex Pair," Aircraft Wake Turbulence and Its Detection, 1971, pp. 305-338. https://doi.org/10.1007/978-1-4684-8346-8_19

[3] Spalart, P. R., “Airplane trailing vortices,” Annual Review of Fluid Mechanics, Vol. 30, 1998, pp. 107-138. https://doi.org/10. 1146/annurev.fluid.30.1.107

[4] Margaris, P., and Gursul, I., "Wing tip vortex control using synthetic jets," Aeronautical Journal, Vol. 110, No. 1112, 2006, pp. 673-681. https://doi.org/10.1017/S0001924000001536

[5] Dghim, M., Ferchichi, M., and Fellouah, H., "Mid-wake wing tip vortex dynamics with active flow control," Experimental Thermal and Fluid Science, Vol. 98, 2018, pp. 38-55. https://doi.org/10.1016/j.expthermflusci.2018.05.011

[6] Crouch, J. D., Miller, G. D., and Spalart, P. R., “Active-control system for breakup of airplane trailing vortices," AIAA Journal, Vol. 39, No. 12, 2001, pp. 2374-2381. https://doi.org/10.2514/2.1244

[7] Crouch, J., “Airplane trailing vortices and their control,”, 2005. https://doi.org/10.1016/j.crhy.2005.05.006.

[8] Gursul, I., and Wang, Z., "Flow control of tip/edge vortices," AIAA Journal, Vol. 56, No. 5, 2018, pp. 1731-1749. https://doi.org/10.2514/1.J056586

[9] Jarrett, P., "FW Lanchester and the Great Divide," Journal of Aeronautical History Paper No, 2014, p. 02.

[10] Chambers, J. R., Partners in Freedom: Contributions of the Langley Research Center to U.S. Military Aircraft of the 1990s, 2000.

[11] Heyson, H., Riebe, G., and Fulton, C., "Theoretical Parametric Study of the Relative Advantages of Winglets and Wing-Tip Extensions," NASA Technical Paper 1020, 1977.

[12] Franklin, A., “AERO Blended Winglets,", Jan 2002. URL http://www.boeing.com/commercial/aeromagazine/aero_17/winglet_ story.html

[13] Cafiero, G., Castrillo, G., Greco, C. S., and Astarita, T., "Effect of the grid geometry on the convective heat transfer of impinging jets," International Journal of Heat and Mass Transfer, Vol. 104, 2017, pp. 39-50. https://doi.org/10.1016/j.ijheatmasstransfer. 2016.08 .003 
[14] Melina, G., Bruce, P. J., Hewitt, G. F., and Vassilicos, J. C., "Heat transfer in production and decay regions of grid-generated turbulence," International Journal of Heat and Mass Transfer, Vol. 109, 2017, pp. 537-554. https://doi.org/10.1016/j. ijheatmasstransfer.2017.02.024

[15] Cafiero, G., Castrillo, G., Greco, C. S., and Astarita, T., "On the effects of square-fractal turbulators on the flow field generated by a synthetic jet actuator," Experimental Thermal and Fluid Science, Vol. 102, 2019, pp. 302-315. https: //doi.org/10.1016/j.expthermflusci.2018.12.005

[16] Goh, K. H., Geipel, P., and Lindstedt, R. P., "Lean premixed opposed jet flames in fractal grid generated multiscale turbulence," Combustion and Flame, Vol. 161, No. 9, 2014, pp. 2419-2434. https://doi.org/10.1016/j.combustflame.2014.03.010

[17] Hurst, D., and Vassilicos, J. C., "Scalings and decay of fractal-generated turbulence," Physics of Fluids, Vol. 19, No. 3, 2007. https://doi.org/10.1063/1.2676448

[18] Mazellier, N., and Vassilicos, J. C., “Turbulence without Richardson-Kolmogorov cascade,” Physics of Fluids, Vol. 22, No. 7, 2010, pp. 1-25. https://doi.org/10.1063/1.3453708

[19] Cafiero, G., Discetti, S., and Astarita, T., "Flow field topology of submerged jets with fractal generated turbulence," Physics of Fluids, Vol. 27, No. 11, 2015. https://doi.org/10.1063/1.4935185.

[20] Nedić, J., Supponen, O., Ganapathisubramani, B., and Vassilicos, J. C., "Geometrical influence on vortex shedding in turbulent axisymmetric wakes,” Physics of Fluids, Vol. 27, No. 3, 2015. https://doi.org/10.1063/1.4913573

[21] Nedić, J., Ganapathisubramani, B., and Vassilicos, J. C., "Drag and near wake characteristics of flat plates normal to the flow with fractal edge geometries," Fluid Dynamics Research, Vol. 45, No. 6, 2013. https://doi.org/10.1088/0169-5983/45/6/061406

[22] Laizet, S., and Vassilicos, J. C., "Fractal space-scale unfolding mechanism for energy-efficient turbulent mixing," Physical Review E - Statistical, Nonlinear, and Soft Matter Physics, Vol. 86, No. 4, 2012. https://doi.org/10.1103/PhysRevE.86.046302

[23] Cafiero, G., Discetti, S., and Astarita, T., "Heat transfer enhancement of impinging jets with fractal-generated turbulence," International Journal of Heat and Mass Transfer, Vol. 75, 2014, pp. 173-183. https://doi.org/10.1016/j.ijheatmasstransfer.2014. 03.049

[24] Cafiero, G., Greco, C. S., Astarita, T., and Discetti, S., "Flow field features of fractal impinging jets at short nozzle to plate distances," Experimental Thermal and Fluid Science, Vol. 78, 2016, pp. 334-344. https://doi.org/10.1016/j.expthermflusci. 2016.06 .009

[25] Gehlert, P., Cafiero, G., and Vassilicos, J. C., "Effect of fractal endplates on the wingtip vortex," AIAA Aerospace Sciences Meeting, 2018, 2018. https://doi.org/10.2514/6.2018-1796

[26] Devenport, W. J., Rife, M. C., Liapis, S. I., and Follin, G. J., “The structure and development of a wing-tip vortex,” Journal of Fluid Mechanics, Vol. 312, 1996, pp. 67-106. https://doi.org/10.1017/S0022112096001929 
[27] Green, S. I., and Acosta, A. J., “Unsteady flow in trailing vortices,” Journal of Fluid Mechanics, Vol. 227, 1991 , pp. 107-134. https://doi.org/10.1017/S0022112091000058

[28] Orloff, K. L., “Trailing vortex wind-tunnel diagnostics with a laser velocimeter,” Journal of Aircraft, Vol. 11, No. 8, 1974, pp. 477-482. https://doi.org/10.2514/3.60371

[29] Willert, C., "Stereoscopic digital particle image velocimetry for application in wind tunnel flows," Measurement Science and Technology, Vol. 8, No. 12, 1997, pp. 1465-1479. https://doi.org/10.1088/0957-0233/8/12/010

[30] Astarita, T., “Analysis of velocity interpolation schemes for image deformation methods in PIV,” Experiments in Fluids, Vol. 45, No. 2, 2008, pp. 257-266. https://doi.org/10.1007/s00348-008-0475-7.

[31] Giordano, R., and Astarita, T., "Spatial resolution of the Stereo PIV technique," Experiments in Fluids, Vol. 46, No. 4, 2009, pp. 643-658. https://doi.org/10.1007/s00348-008-0589-y

[32] Jeong, J., and Hussain, F., “On the identification of a vortex,” Journal of Fluid Mechanics, Vol. 285, 1995 , pp. 69-94. https://doi.org/10.1017/S0022112095000462

[33] Terashima, O., Sakai, Y., and Nagata, K., "Simultaneous measurement of velocity and pressure in a plane jet: Development of well-Arranged combined probe and estimation of turbulent energy budget," Experiments in Fluids, Vol. 53, No. 4, 2012, pp. 1149-1164. https://doi.org/10.1007/s00348-012-1351-z 